\title{
Medición del desarrollo humano a escala territorial: metodología y su aplicación a los casos de Argentina y México
}

\section{Measurement of human development at a regional scale: its methodology and application to the cases of Argentina and Mexico}

María Albina Pol*

\begin{abstract}
This work presents the results from a research whose starting point is the consideration of human developments as a process of territorial nature; the accomplishments of people are determined by the surrounding wherein they commonly live. This states the need to advance in the formulation of theoretical-methodological approaches that include the spatial dimension of development. On this basis, and from corroborating the scarce capacity of HDI-UNDP to give an account of the unequal distribution of capabilities at sub-national scale in Latin American countries, we constructed the new measure, which adjusted to the UNDP methodology, presents greater territorial sensibility. The exercise of application of the index of territorial human development to Argentina and Mexico allowed drawing some conclusions on its validity.
\end{abstract}

Keywords: human development, territory, indicators.

\section{Resumen}

Este trabajo expone los resultados de una investigación cuyo punto de partida es la consideración del desarrollo humano como proceso de naturaleza territorial, los logros de las personas los determina el entorno en el que se desarrollan cotidianamente. Esto plantea la necesidad de avanzar en la formulación de encuadres teórico-metodológicos que incluyan la dimensión espacial del desarrollo. Sobre esa base, y a partir de comprobar la escasa capacidad del IDH-PNUD para dar cuenta de la desigual distribución de las capacidades a escala subnacional en países de América Latina, se construyó una nueva medida que, ajustada a la propuesta metodológica del PNUD, presenta una mayor sensibilidad territorial. El ejercicio de aplicación del índice de desarrollo humano territorial a Argentina y México permitió extraer algunas conclusiones sobre su validez.

Palabras clave: desarrollo humano, territorio, indicadores.

* Universidad Nacional de Cuyo, Argentina. Correo-e: albinapol@yahoo.com.ar. 


\section{Introducción}

En las nuevas concepciones acerca del proceso de desarrollo, surgidas hace algunas décadas pero notablemente fortalecidas durante los últimos ańos, el territorio aparece revalorizado en tanto espacio de configuración de las dinámicas de crecimiento económico y mejora en el bienestar de una población.

Desde esa perspectiva, es posible concebir al desarrollo humano, paradigma cuyos supuestos básicos reposan en el enfoque de las capacidades humanas, como un proceso de naturaleza territorial, en la medida en que las oportunidades al alcance de las personas y su potencial expansión aparecerían condicionadas por el entorno donde los individuos se desarrollan cotidianamente. Esto lleva a sostener la importancia de avanzar en la formulación de encuadres teóricos y metodológicos que incluyan la dimensión espacial y faciliten la aproximación al patrón de distribución territorial de las capacidades humanas al interior de los ámbitos nacionales.

El índice de desarrollo humano (IDH) propuesto por el Programa de las Naciones Unidas para el Desarrollo (PNUD) constituye uno de los intentos más reconocidos de operacionalización de las capacidades humanas. El IDH se creó con el objetivo de aproximar los progresos generales de un conjunto de países en las dimensiones esenciales del desarrollo humano y para las que existieran datos disponibles y susceptibles de ser comparados.

Siguiendo con las consideraciones que señalan la importancia de la dimensión territorial en la configuración de los logros al alcance de las personas, cabe indagar acerca de la sensibilidad del IDH para dar cuenta de la distribución de las capacidades entre entidades espaciales de escala subnacional. En otras palabras, interesa examinar si ese índice es una medida válida para reflejar las diferencias en el acceso a oportunidades en las distintas unidades en las que se organiza un país.

Como primera aproximación, el análisis de las aplicaciones realizadas por los equipos del PNUD en América Latina arroja evidencias acerca de la escasa sensibilidad del IDH para reflejar disparidades en los territorios nacionales, y muestran que dicha sensibilidad disminuye a medida que los países adquieren un mayor nivel de desarrollo.

Partiendo de esa constatación, el artículo tiene por objeto exponer sintéticamente el proceso de investigación que dio como resultado la formulación de una nueva medida que, sin pretender reemplazar al IDH en tanto herramienta con valor comparativo entre naciones, busca captar de manera más precisa las disparidades territoriales del desarrollo humano.

El procedimiento metodológico consistió en someter el IDH-PNUD a una serie de análisis para identificar sus limitaciones en relación con el 
objeto de estudio y, a partir de ello, incorporar algunas modificaciones centradas en las variables que lo integran. Respetando la estructura conceptual y metodológica del IDH-PNUD, se obtuvo un indice de desarrollo humano territorial (IDHT), fundamentado en el supuesto de alcanzar mayor pertinencia en aquellos países de América Latina que han logrado un nivel de desarrollo relativamente más elevado. El artículo está organizado de acuerdo con esa lógica; después de esbozar brevemente los lineamientos conceptuales, se describe el procedimiento seguido en la construcción del IDHT, para luego presentar y examinar los resultados de su aplicación en Argentina y México y extraer algunas conclusiones sobre su validez.

\section{La naturaleza territorial del desarrollo humano}

El desarrollo humano consiste en ampliar las libertades de las personas, en generar cada vez más opciones de vida entre las cuales elegir. Esta perspectiva tiene sus raíces en el enfoque de las capacidades humanas propuesto por Amartya Sen (2000), que se distancia de las corrientes tradicionales en el sentido de enfatizar la eliminación de los obstáculos que dificultan o impiden las posibilidades de ser o actuar de acuerdo con los propios objetivos, y no en la mera acumulación de recursos materiales. Lo que se valora son las posibilidades a las que efectivamente pueden acceder las personas en un determinado tiempo y lugar.

Esa relatividad histórico-espacial del desarrollo humano implica que las capacidades de decisión y elección están condicionadas por el entorno inmediato. El lugar donde viven, las relaciones que establecen y los recursos disponibles en el medio, son factores determinantes del espectro y tipo de opciones que las personas tienen a su alcance. Por tanto, las oportunidades, su valoración y selección difieren según las circunstancias que las rodean.

En este punto, la concepción del desarrollo como expansión de las libertades se enlaza con la noción del desarrollo en tanto proceso territorialmente localizado, según el cual el entorno espacial es una construcción social dinámica resultado de las múltiples, complejas y particulares relaciones que se establecen entre factores de diversa índole (Rofman, 2006).

La especificidad del territorio en que viven lleva a las personas a establecer determinado tipo de vínculos entre sí y con las instituciones más próximas, a ejercer sus libertades y realizar proyectos que a su vez modifican ese entorno inmediato (Barreiro, 2007). El entramado territorial es el ámbito en el que las libertades se amplían o limitan, es el espacio donde el proceso de desarrollo humano tiene lugar.

Estas consideraciones llevan a sugerir que el conocimiento de la distribución espacial de las capacidades humanas, constituye un primer paso 
esencial para identificar territorios y áreas prioritarios hacia los cuales dirigir estrategias que tiendan a igualar las oportunidades de las personas. Contar con información que permita discriminar las diferentes situaciones en las que se encuentran los territorios facilita el diseño, gestión y seguimiento de las políticas públicas. Este desafío adquiere especial relevancia en los países de América Latina donde la desigualdad, en sus diferentes dimensiones, aparece como una característica estructural. La naturaleza territorial del desarrollo humano exige entonces examinarlo en entidades geográficas específicas.

Numerosos son los debates conceptuales y ejercicios empíricos orientados a avanzar en la operacionalización de las capacidades consideradas fundamentales para el desarrollo de las personas. Uno de los intentos más aceptados por los medios políticos y técnicos ha sido el IDH, medida que el PNUD propuso en su primer informe mundial con la intención de tener una idea resumida del nivel de logro en desarrollo humano de cada país. El IDH, usado internacionalmente y diseñado para comparar 178 países, se construyó sobre la base de un perfil de desarrollo promedio y para realidades muy diversas en términos de disponibilidad de estadísticas. En consecuencia, resulta menos útil cuando se utiliza para mirar al interior de países con un nivel de desarrollo comparativamente más elevado.

\section{Análisis de sensibilidad territorial de los indicadores que componen IDH-PNUD}

Aunque las necesidades de las personas son múltiples y cambian a lo largo del tiempo, existen algunas condiciones básicas que son comunes a todas las sociedades y en todo tiempo: tener una vida larga y saludable; poseer los conocimientos necesarios para comprender y relacionarse reflexivamente con el entorno social y contar con ingresos suficientes para acceder a un nivel de vida decente. Éstas son las dimensiones esenciales que se pretenden captar por medio del IDH.

La delimitación de indicadores específicos para aproximar cada una de las dimensiones en un conjunto heterogéneo de países, fuerza a compatibilizar los criterios de validez con la disponibilidad de datos estadísticos, aspecto en el que se centran algunas de las principales críticas dirigidas al IDH. De ahí que el mismo se plantee como una medida abierta y flexible susceptible de modificaciones y adaptaciones para mejorar su ajuste a las capacidades que intenta evaluar.

Los logros en salud se miden con la esperanza de vida al nacer. Los avances educativos se evalúan combinando la tasa de alfabetización de adultos y la tasa bruta de matrícula combinada primaria, secundaria y superior. El logro en la dimensión ingreso se calcula a partir del produc- 
to interno bruto (PIB) per cápita ajustado por paridad de poder adquisitivo en dólares.

Dotar al IDH-PNUd de mayor validez y, al mismo tiempo, de sensibilidad para reflejar la desigual distribución de las capacidades entre unidades subnacionales requiere, en primera instancia, centrar el análisis en sus variables componentes. Es necesario comenzar indagando si cada uno de los indicadores que integran el índice sintético constituye una medida capaz de mostrar la forma en que se distribuyen los logros en un país determinado.

Para alcanzar ese objetivo se seleccionó un conjunto de países de América Latina con disponibilidad de datos desagregados. ${ }^{1}$ Mediante el análisis de variabilidad, basado en el cálculo e interpretación de la desviación estándar y el rango de las series de datos, se obtuvieron algunas conclusiones referidas a la sensibilidad territorial de las variables originales.

Los resultados permitieron constatar que la distribución de algunos indicadores del IDH-PNUD se hace significativamente homogénea a medida que el país adquiere cierto grado de desarrollo. Es decir, al mejorar las condiciones de vida de una población las medidas pierden capacidad para reflejar las diferencias al interior del territorio nacional.

La homogeneización de las variables del IDH-PNUD, no sólo en términos desagregados sino incluso cuando se comparan los totales nacionales para diferentes países, ha sido señalada por autores como Kelley (1991) y Qizilbash (2002). La metodología propuesta por el PNUD resulta más adecuada para discriminar la situación de los países que arrojan valores del IDH bajos que la de aquéllos que presentan valores medios o altos. ${ }^{2}$ Estas apreciaciones se ilustran por medio del análisis de variabilidad. ${ }^{3}$

De manera específica, se comprobó cómo se distribuye la esperanza de vida al nacer a escala subnacional. La desviación estándar de este indica-

${ }^{1}$ Argentina, Brasil, Chile y México. El desglose de los datos se realizó considerando las cifras más recientes disponibles en cada país.

${ }^{2}$ Cuestión también reconocida por el pNUD (2006: 264).

${ }^{3} \mathrm{El}$ análisis de variabilidad se realizó a partir del cálculo de los valores de la desviación estándar y el rango de las series de datos correspondientes a cada una de las variables para Argentina (provincias), Brasil (estados), Chile (regiones) y México (estados), según los últimos datos disponibles en cada caso. Las fuentes consultadas fueron:

Argentina: INDEC, Ministerio de Salud y Ambiente, Dirección de Estadísticas e Información de Salud, Estadísticas Vitales. FLAcso Educación, Ministerio de Educación, Ciencia y Tecnología, Dirección Nacional de Información y Evaluación de la Calidad Educativa.

Brasil: Projeto IBGE/Fundo de População das Naçōes Unidas-UnFpa/brasil (bra/98/P08), Sistema Integrado de Projeçóes e Estimativas. Ministerio de Salud/Funasa/Cenepi-Sistema de Informaçóes sobre Mortalidade-IBge, Diretoria de Pesquisas, Pesquisa Nacional por Amostra de Domicílios 2003.

Chile: INE, Departamento de Estadísticas e Información de Salud, Ministerio de Salud, Compendio de Estadísticas Vitales. Ministerio de Educación: www.mineduc.cl/biblio/novedades/doc/ Indicadores_para_la_educacion.pdf.

México: INegi, pNUd Informe Nacional sobre Desarrollo Humano México 2002, Conapo. Dirección General de Planeación, Programación y Presupuesto. Secretaría de Educación Pública. 
dor oscila entre 2.1 en Brasil y 0.9 en Chile, para los ańos 2000 y 2001, respectivamente. Esta diferencia refuerza el supuesto de que a mayor nivel de desarrollo humano menor sensibilidad de la variable para reflejar disparidades territoriales. Brasil es uno de los países más desiguales en términos económicos y sociales de la región, lo que justificaría una relativamente heterogénea distribución de la esperanza de vida entre sus unidades territoriales. Chile, en cambio, se ubica entre los países más equitativos y con mayor desarrollo humano de América Latina.

$\mathrm{Al}$ analizar la distribución espacial de la tasa de alfabetización de adultos aparece nuevamente una clara disparidad entre los países que han conseguido un mayor nivel de desarrollo humano y los que presentan un menor grado de avance relativo. La alfabetización de adultos ha adquirido carácter prácticamente universal en Argentina y Chile, lo que queda constatado en los valores de la desviación estándar - 1.9 y 2.4 , respectivamente. En 2001 la tasa de alfabetización de Argentina era de 97.4\% y la de Chile de 96 por ciento.

Un panorama marcadamente distinto se configura en países como Brasil y México que, según la clasificación del PNUD, arrojan un nivel de desarrollo humano inferior al de Chile y Argentina. En las series de datos obtenidas, la desviación estándar de la tasa de alfabetización de adultos alcanzaba 8.6 en Brasil y 4.8 en México. En el primer caso, la medida indica la alta heterogeneidad con que se distribuye la alfabetización entre los estados. Para el año 2003 el indicador registraba un valor nacional de $88.4 \%$, y valores extremos de 71.6 y $95 \%$. En México, que presentaba en 2004 una tasa de alfabetización de $91.5 \%$, los valores mínimo y máximo a nivel estadual fueron de 81.2 y 98.2 por ciento.

En lo relativo a la tasa bruta de matriculación combinada, la obtención desagregada de la variable para cada uno de los países presentó una mayor complejidad, dadas las diferencias entre los sistemas educativos nacionales. Como consecuencia, se tomó la decisión de analizar la sensibilidad territorial del indicador restringiendo su amplitud a los niveles de primaria y secundaria. Los resultados muestran que la tasa de matrícula escolar se distribuye de manera desigual en todos los territorios, sin ajustarse a un patrón asociado al nivel de desarrollo de cada país. En otras palabras, no se repite la pauta que indica que a mayor desarrollo relativo mayor homogeneidad espacial en la distribución de la variable. Utilizando los datos para ilustrar esta conclusión, se observa que, según el valor de la desviación estándar, el país que presenta una distribución más desigual es Argentina (8.3), seguido por Brasil (7), México (4.8) y Chile (3). En cuanto a los rangos de la variable, las diferencias entre el máximo y el mínimo valor oscilan entre 39 puntos porcentuales para las provincias argentinas y 12 para las regiones de Chile. 
Finalmente, el indicador utilizado en el IDH-PNUD para medir la dimensión nivel de vida (PIB per cápita), no se sometió a análisis de sensibilidad territorial por no contar con información estadística suficiente para su elaboración a escala desagregada, pero asimismo porque no se considera una medida válida para reflejar la capacidad que con él se pretende evaluar. ${ }^{4}$

En síntesis, el análisis de sensibilidad territorial aplicado a las variables que componen el IDH-PNUD arroja las siguientes conclusiones. La esperanza de vida al nacer resulta una medida poco sensible a las disparidades territoriales cuando los países han alcanzado un nivel de desarrollo relativamente alto. Esto sugiere la necesidad de reemplazarla por otro indicador más efectivo cuando se mide la forma en que se distribuyen las capacidades en salud entre las unidades subnacionales. En relación con el componente educación, la tasa de alfabetización de adultos presenta las mismas características que la esperanza de vida al nacer, se torna espacialmente homogénea en países que presentan un mayor grado de avance, dejando sin sentido su incorporación a un índice territorial. Los datos referidos a la matrícula escolar no dan información significativa para medir las heterogeneidades territoriales en términos de logros educativos, esta conclusión lleva a sugerir el reemplazo del indicador o bien la incorporación de otra variable que lo complemente y permita precisar la información por él aportada.

\section{Búsqueda, selección y evaluación de variables alternativas}

Con base en las conclusiones derivadas del análisis de sensibilidad territorial, la segunda etapa de la metodología se centró en la búsqueda, selección y evaluación de indicadores disponibles que, además de validez conceptual, presentaran una mayor adecuación para medir las disparidades espaciales en cada una de las dimensiones del IDH. Identificados los ajustes a realizar al IDH-PNUD, el resultado de esta fase es la elaboración de un índice de desarrollo humano territorial (IDHT).

\subsection{Búsqueda y selección de nuevas variables}

En lo relativo a la dimensión salud, la consulta de documentos elaborados por la Organización Mundial de la Salud (oms), el Banco Mundial y la Comisión Económica para América Latina y el Caribe (CEPAL), arrojó

\footnotetext{
${ }^{4}$ Diversos autores (Nordhaus y Tobin, 1973; Hicks y Streeten, 1979; Morris, 1979; Ram, 1982; PNUD, 1990; Slottje, 1991; Ul Haq, 1995; Sen, 2000; Stanton 2007, entre otros) han señalado los problemas conceptuales del PIB en tanto aproximación al bienestar económico de los habitantes de un país.
} 
como resultado la selección de cinco variables susceptibles de reemplazar a la esperanza de vida al nacer: 1) tasa de mortalidad infantil, 2) tasa de mortalidad en menores de cinco años, 3) tasa de mortalidad materna, 4) cantidad de médicos por 10,000 habitantes y 5) número de camas por 10,000 habitantes.

La tasa de mortalidad infantil es uno de los indicadores que mayor atención recibe de las autoridades nacionales, los organismos internacionales y la sociedad en general. Esto se debe a que refleja, de manera relativamente precisa, el grado de desarrollo alcanzado por una población al estar muy relacionada con el acceso a servicios básicos de salud, la calidad de los mismos, el nivel educativo de los progenitores, el estado nutricional de la madre y el niño, entre otros aspectos. La tasa de mortalidad en menores de cinco años es una ampliación de la tasa de mortalidad infantil y se ha incluido como uno de los indicadores para dar seguimiento a los Objetivos de Desarrollo del Milenio.

Las muerte materna constituye otro importante indicador del estado de salud de una población, es una expresión de la inequidad y el rezago social, así como un reflejo de los problemas de cobertura y calidad de los servicios de salud, estado nutricional, nivel educativo, etc. De ahí que este indicador también se incluya en el monitoreo de los Objetivos de Desarrollo del Milenio.

La disponibilidad de recursos humanos y de infraestructura sanitaria básica también se pueden considerar indicadores de las posibilidades de las personas para acceder a los servicios de salud y, por tanto, de sus capacidades para una vida sana y decorosa. Una de las variables disponibles para medir el primero de esos aspectos es la cantidad de médicos por 10,000 habitantes. La distribución de estos recursos muestra, en general, la existencia de brechas importantes en la disponibilidad de profesionales entre las diferentes jurisdicciones. Sin embargo, se debe tener en cuenta que muchas instituciones, en especial las asentadas en centros urbanos, prestan servicios a usuarios procedentes de otras entidades.

Con el fin de evaluar la disponibilidad, acceso y distribución de la prestación de servicios de salud, es preciso contar con una serie de indicadores o bien con un indicador compuesto de infraestructura sanitaria. En la actualidad no se dispone de tales datos en la mayoría de los países. El número de camas hospitalarias disponibles por 10,000 habitantes es una de las pocas medidas relacionadas con dicho componente, accesible en un gran número de países. La utilización de este indicador también se encuentra limitada por la concentración espacial de las infraestructuras sanitarias.

En síntesis, las variables referidas a la mortalidad en los primeros años de vida y a la mortalidad materna tienen una significativa relevancia como 
medidas del nivel de salud general de una población. Las primeras también arrojan una fuerte asociación con la esperanza de vida, lo que garantiza su consistencia con la base conceptual original. Los indicadores de disponibilidad de recursos sanitarios, además de que son medidas poco precisas, muestran limitaciones derivadas de la concentración territorial de los servicios y de la escasez de información para su elaboración.

Para identificar los indicadores referidos al aspecto educación se revisaron fuentes elaboradas por la UnEsco, el Banco Mundial y las bases de datos Badeinso de la cepal y siteal de la Organización de Estados Iberoamericano (OEI), ello derivó en la selección de tres indicadores que se considera posibilitan una aproximación más precisa a las capacidades disponibles en las entidades subnacionales: 1) nivel educativo en personas de 25 años y más, 2) años promedio de escolaridad en personas de 25 años y más y 3) tasa de asistencia escolar.

Las variables de nivel educativo y años promedio de escolaridad, al igual que la tasa de alfabetización de adultos, se consideran indicadores de efecto, ya que aportan información acerca de los conocimientos acumulados en una determinada población. Sin embargo, para dar cuenta de los logros educativos en países que han alcanzado un nivel relativamente más elevado de desarrollo, los indicadores seleccionados presentan una mayor pertinencia que la variable de alfabetización de adultos. El aspecto más importante, vinculado a la conceptualización del desarrollo como proceso de ampliación de las opciones de las personas, es que un mayor nivel de educación aumenta el rango de oportunidades de empleo, facilitando su integración en la vida de la comunidad (Herrero et al., 2004).

La utilización de la variable años promedio de escolaridad se sugirió en el segundo informe mundial del PNUD, ${ }^{5}$ pero se sustituyó del IDH debido a la dificultad que plantea su obtención para todos los países considerados en la clasificación internacional. En este trabajo dicho indicador se sometió a análisis con el propósito de determinar su posibilidad de cálculo desagregado en los países de la región.

La tasa de asistencia escolar, así como las tasas de matrícula, se denominan indicadores de cobertura dado que captan la capacidad de retención del sistema. Su construcción requiere datos que aporten información sobre la cantidad de personas que, en un momento dado, asisten a los distintos niveles de la educación formal. Compatibilizar esta variable para diferentes países requiere una cuidadosa revisión de la estructura de los

\footnotetext{
${ }^{5}$ Con la incorporación, a partir de 1991, de la variable años promedio de escolaridad en el IDH se buscó completar la información aportada por la tasa de alfabetización reconociendo, con ello, la importancia de los mayores niveles de educación y, principalmente, con el propósito de discriminar y caracterizar la situación educativa de los países que se encuentran en los primeros puestos del ranking, en particular las economías industrializadas (PNUD, 1991: 15).
} 
sistemas educativos y la utilización de grandes grupos de edad que incluyan a todas las personas que asistan en un momento determinado.

En conclusión, medir los logros educativos de una determinada población debe contemplar dos aspectos: las capacidades efectivas, consecuencia de los conocimientos previamente adquiridos, lo que en el índice original se mide mediante la tasa de alfabetización de adultos, y la capacidad del propio sistema para brindar oportunidades educativas a sus habitantes, lo que en el IDH-PNUD se evalúa por medio de la tasa de matrícula combinada. De acuerdo con ese planteamiento, los dos indicadores de efecto susceptibles, en términos conceptuales, de reemplazar a la tasa de alfabetización para la configuración del nuevo índice, son el nivel educativo y los años promedio de escolaridad. En cuanto a los indicadores de cobertura, el análisis aplicado sugiere evaluar el mantenimiento de la matrícula combinada, realizando algunos ajustes, o bien su reemplazo por la tasa de asistencia escolar.

Para seleccionar las variables asociadas con la dimensión nivel de vida del IDH se consultaron fuentes elaboradas por el Banco Mundial, la base de datos Bandeinso, las definiciones de indicadores de Naciones Unidas y fuentes estadísticas nacionales.

La medición territorialmente desagregada del componente económico del IDH plantea el doble desafío de evaluar el nivel de ingresos disponible en cada entidad espacial y el grado de concentración o dispersión con que el mismo se distribuye. Sólo la captación de ambos elementos permite obtener un panorama más o menos preciso de las posibilidades reales de acceso a recursos materiales por parte de una determinada población. Con el objetivo de reflejar el primero de esos aspectos se seleccionó la variable ingreso per cápita de los hogares; para el segundo se identificaron tres indicadores: 1) tasa de desocupación, 2) tasa de subocupación horaria y 3) porcentaje de personas en la línea de pobreza.

$\mathrm{Al}$ analizar las aplicaciones del IDH a escala subnacional realizadas por los equipos del PNUD, se observó que el PIB per cápita generalmente se reemplaza con algún indicador de ingreso que, como se construyen actualmente, tampoco resultan medidas óptimas para capturar las posibilidades reales de acceso de las personas a los recursos materiales, ya que no incluyen todos los aspectos determinantes. A pesar de ello, brindan una representación más válida que el PIB per cápita, puesto que se refieren a variables medidas a partir de la realidad económica de las personas, lo que las sensibiliza a la forma real de la distribución del fenómeno (PNUD, 1996: 57).

La importancia de considerar en la medición del desarrollo humano la desigual distribución de los logros, en especial en lo que se refiere al 
componente ingreso, ${ }^{6}$ se reconoce desde el primer informe mundial (PNUD, 1990: 38$)^{7}$ y su análisis se encuentra ampliamente incorporado en los informes nacionales.

El efecto de la desigualdad en la distribución de la renta se puede incorporar al IDH aplicando diferentes metodologías. Una de ellas consiste en realizar ajustes a los resultados una vez obtenido el índice para cada unidad subnacional, mediante el cálculo de coeficientes de concentración (PNUD, 2000, 2004a) o de medias generalizadas (PNUD, 2002). Una segunda opción es agregar a la composición del índice uno o más indicadores que den alguna aproximación a la forma en que se distribuye el ingreso en cada unidad espacial, como aquéllos referidos a incidencia de la pobreza o a las dificultades de acceso al mercado laboral (PNUD, 2005).

En síntesis, la tercera dimensión del IDH es la más difícil de medir, su cálculo a escala territorial debe contemplar la evaluación del nivel de ingresos disponible en cada territorio y la forma en que el mismo se distribuye. De entre los indicadores susceptibles de ser incorporados al IDHT, el ingreso per cápita de los hogares aparece como la variable más idónea. La consideración de la desigualdad se puede incorporar a la medición realizando correcciones posteriores al cálculo del índice, o bien agregando al indicador de ingresos alguna medida que refleje la concentración o dispersión de la renta en cada territorio.

\subsection{Evaluación de las variables seleccionadas}

Una vez que se constató la disponibilidad de datos territorialmente desagregados para las variables identificadas, a los indicadores se aplicaron diferentes procedimientos analíticos para establecer los ajustes necesarios para elaborar el nuevo índice.

- Salud. Sobre la base de los datos desagregados de Argentina, Brasil, Chile y México, las variables escogidas se sometieron a dos tipos de análisis: determinación del grado de correlación con la esperanza de vida al nacer, a fin de contrastar su validez conceptual con la de la variable original; e identificación, a través de medidas de variabilidad, de la capacidad de cada indicador para discriminar los logros a escala subnacional.

${ }^{6} \mathrm{Si}$ bien la desigualdad en la distribución de los indicadores de salud y educación puede ser considerable, el hecho de que éstas sean variables acotadas limita significativamente el posible grado de disparidad.

${ }^{7}$ En los informes hasta 1994 se muestra cómo se alteraría el desempeño de los países si se corrigiera el ingreso mediante el coeficiente de Gini. A pesar de la importancia de este tema, la ausencia de datos confiables, completos y actualizados para la mayor parte de los países ha evitado que este tipo de consideraciones se incorpore permanentemente al cálculo del índice. 
- Educación. Comprobada la escasa homogeneidad de las estadísticas educativas entre los países de la región, se procedió a evaluar distintas variables de forma similar al procedimiento seguido para los indicadores de la dimensión salud.

- Nivel de vida. Partiendo de la escasa validez del pib per cápita, se fundamentan los indicadores con los cuales se reemplaza.

En lo que respecta al tema de salud, el rastreo y análisis de las fuentes estadísticas llevó a identificar ciertas restricciones en la información requerida para el cálculo de algunos de los indicadores seleccionados. De entre las variables de mortalidad, la que presenta mayores dificultades de registro es la tasa de mortalidad materna. Las muertes maternas son particularmente difíciles de medir ya que, en buena parte de los países, hay problemas de subregistro debido a la deficiente clasificación de las causas de muerte. Por otra parte, los datos presentan limitaciones al no considerar los nacimientos producidos fuera de los servicios del sistema sanitario.

Los indicadores de recursos humanos y materiales también presentan deficiencias de registro que se suman a la mencionada concentración espacial de las infraestructuras sanitarias. No todos los países elaboran este tipo de estadísticas, los criterios de registro varían de un entidad a otra y, además, existen problemas en cuanto a la sistematización y actualización de los datos.

A pesar de estas restricciones, todos los países considerados -excepto Chile, para el que no fue posible obtener datos sobre recursos sanitarios en el ámbito regional- cuentan con información desagregada para el cálculo de las variables seleccionadas. De ahí que el análisis de correlación con la esperanza de vida al nacer se aplicara a todas ellas. El supuesto que se sostiene afirma que a mayor correlación más correspondencia con el concepto que se pretende medir, dada la alta validez de la variable original. ${ }^{8}$

Según los valores arrojados por la correlación, la variable que mayor asociación presenta con la esperanza de vida es la tasa de mortalidad en menores de cinco años, que sólo en el caso de Chile alcanza un valor de -0.51 , pero para el resto de los países se coloca entre -0.80 y -0.90 . El grado de correlación de esta variable con la esperanza de vida es levemente superior al de la tasa de mortalidad infantil.

La tasa de mortalidad materna alcanza en su correlación con la esperanza de vida una significancia considerablemente más baja que la tasa de mortalidad en menores de cinco años. En los extremos se colocan Argentina con un coeficiente de -0.62 y Chile con -0.14 . Esta disparidad en las

\footnotetext{
${ }^{8}$ Los cálculos se realizaron tomando como base las series desagregadas disponibles en cada país para el año más reciente, y se correlacionó cada variable con la esperanza de vida al nacer correspondiente a ese mismo año.
} 
correlaciones de países con perfiles de desarrollo similares, estaría reflejando, entre otros aspectos, los problemas de registro de la variable.

Los indicadores de recursos humanos y materiales, cantidad de médicos y de camas por 10,000 habitantes arrojan una correlación poco significativa con la esperanza de vida al nacer (entre 0.18 y 0.56 el primero y entre 0.15 y 0.54 el segundo). La débil asociación y los problemas de registro llevan a desestimar la consideración de estas variables como medidores de la dimensión salud del IDHT.

Una vez que se identificó la elevada significancia de la tasa de mortalidad infantil y la tasa de mortalidad en menores de cinco ańos, y con el objetivo de determinar su sensibilidad para captar las disparidades territoriales, se realizó el cálculo de la desviación estándar y el rango de las series de datos.

Los valores de las desviaciones estándar brindan información que aporta a la mayor significancia de la tasa de mortalidad en menores de cinco ańos como indicador susceptible de reemplazar a la esperanza de vida al nacer. El valor más alto de la desviación se observa en el caso de Brasil (11.53) y el más bajo en Chile (1.14), diferencia que da cuenta de las desigualdades en el nivel de desarrollo alcanzado por las unidades espaciales en cada uno de esos países.

El análisis de dispersión territorial se completa con la observación de los valores extremos y las diferencias porcentuales entre los mismos. Los valores máximo y mínimo para cada serie de datos refuerzan la fundamentación de utilizar la tasa de mortalidad en menores de cinco años. Sólo en el caso de Brasil la brecha porcentual entre los valores extremos es levemente mayor en la tasa de mortalidad infantil (285\% frente a $273 \%)$. El país que arroja la menor diferencia es Chile (42\%), sin olvidar que la división administrativa utilizada para la desagregación de los datos son las regiones, probablemente si se utilizaran unidades de menor amplitud la diferencia tendería a aumentar.

En síntesis, tanto el análisis de correlación con la variable original como de sensibilidad para reflejar las disparidades territoriales, permiten concluir que la tasa de mortalidad infantil en menores de cinco años se presenta como el indicador más pertinente para reemplazar a la esperanza de vida al nacer en la dimensión salud del IDHT.

Con el objetivo de analizar la posibilidad de cálculo a escala subnacional de las variables seleccionadas para el tema educación del IDHT, se realizó un exhaustivo rastreo de información entre las fuentes nacionales disponibles, que dio como resultado la existencia de fuertes discrepancias y limitaciones en las estadísticas educativas de los países de la región.

Para dar cuenta de los logros educativos a escala subnacional se requiere, por un lado, incorporar al índice alguna medida que refleje el capital 
humano acumulado en el territorio, en reemplazo de la tasa de alfabetización de adultos. Y por otro, evaluar la sustitución o ajuste de la tasa de matrícula combinada para aproximar de manera más precisa las oportunidades efectivas de acceso a la educación y su distribución espacial.

Entre las variables seleccionadas, sólo fue posible obtener de forma desagregada para todos los países considerados la correspondiente al nivel educativo, medido a partir de la cantidad de personas de 25 años y más con secundaria completa o más. Con base en esa constatación, se realizó un análisis de variabilidad para comparar la sensibilidad territorial de ese indicador respecto de la tasa de alfabetización. El objetivo del análisis es aportar evidencia empírica que justifique la sustitución de la alfabetización por un indicador más exigente en términos de logros educativos.

El cálculo de la desviación estándar arrojó, en términos generales, una considerable mayor dispersión de la variable de nivel educativo frente a la tasa de alfabetización. Resulta interesante destacar el caso de Brasil, único país en que la desviación de la variable original es superior a la del nivel educativo, 8.62 y 6.17, respectivamente. Es posible que esto evidencie que la tasa de alfabetización de adultos todavía funciona como un indicador válido para discriminar los logros educativos de las unidades territoriales en ese país, dado el nivel de desarrollo que presenta. Para los otros tres casos la desviación estándar del nuevo indicador alcanza valores superiores a los de la tasa de alfabetización de adultos.

Los valores extremos de cada serie de datos muestran la gran amplitud de recorrido del indicador de nivel educativo. Los valores superiores arrojados por esta variable permiten deducir que se trata de una medida más exigente que la tasa de alfabetización. En otras palabras, los datos indican que en todos los países hay un significativo margen de acción en materia de ampliación de oportunidades para facilitar el acceso a mayores niveles de educación.

Estos resultados llevan a concluir acerca de la pertinencia de reemplazar la tasa de alfabetización de adultos por el porcentaje de personas de 25 años y más que ha completado al menos la secundaria. Esta última variable, además de presentar una mayor validez para dar cuenta de los logros educativos en los países más avanzados, se distribuye de manera notablemente más desigual que la tasa de alfabetización de adultos.

En lo referente al segundo objetivo de medición del componente educativo, evaluar las posibilidades efectivas de acceso a la educación, se pudo confirmar que una de las variables básicas disponibles es la tasa de matrícula para los distintos niveles, lo que justificaría su permanencia en el IDHT. En cuanto al indicador considerado para su reemplazo, la tasa de asistencia escolar, no fue posible obtener información territorialmente desagregada para su construcción en todas las entidades analizadas. 
En definitiva, contar con indicadores educativos homogéneos para los países de la región es una tarea compleja. La tasa de matrícula combinada restringida a los niveles de primaria y secundaria, aunque aporte una aproximación indirecta a la dimensión y presente algunos problemas de registro, sigue siendo una de las variables básicas disponibles a escala desagregada, lo que justifica su permanencia en el índice. Por otra parte, se estima conveniente incorporar, en reemplazo de la tasa de alfabetización de adultos, la variable de nivel educativo en personas de 25 años y más. Es de esperar que esta modificación, fundamentada en la escasa validez de la alfabetización como reflejo del capital humano instalado en el territorio y, por tanto, en la importancia de incorporar un indicador más exigente en términos educativos (Murray 1991: 6), genere un efecto considerable en los resultados arrojados por el índice.

La selección de los indicadores vinculados a la dimensión nivel de vida del IDHT busca configurar un subíndice compuesto que, además de evaluar las posibilidades de acceso a recursos materiales, nos aproxime a la forma en que se distribuye la renta en cada territorio. El primer aspecto debería evaluarse por medio de un subíndice sintético que incorpore indicadores relativos no sólo a las remuneraciones monetarias percibidas por los individuos, sino también a un conjunto de aspectos no monetarios fuertemente vinculados a la disponibilidad y titularidad de los recursos. Sin embargo, no se cuenta con información estadística suficiente, homogénea y confiable para construir ese tipo de medidas.

Dadas estas limitaciones, el PNUD incorpora al IDH el PIB per cápita porque no cuenta con otra variable más pertinente para todas las unidades consideradas. Sin embargo, al acotar la medición a los países de América Latina, es posible disponer de un indicador que, aun con limitaciones, presenta un mayor grado de validez: el ingreso per cápita de los hogares.

Según se pudo comprobar, prácticamente todos los países de la región cuentan con fuentes que revelan datos sobre el ingreso de las personas y los hogares, aunque en la mayoría de los casos esta información no cubre la totalidad del territorio nacional, en general se refiere a la población que reside en zonas urbanas. Sin embargo, tratándose de países con una alta concentración demográfica en ese tipo de áreas, la cobertura poblacional de las encuestas por muestreo oscila entre 60 y $80 \%$ del total. Si bien se entiende que el ingreso per cápita, por las características de la medida, ${ }^{9}$ continúa siendo un indicador débil para evaluar las oportunidades efectivas por parte de todas las personas que residen en una determinada entidad, el supuesto es que alcanza mayor validez que el PIB per cápita. La disponibilidad de información y el mayor grado de validez han per-

${ }^{9}$ Excluye del cálculo a las poblaciones residentes en las denominadas zonas rurales. 
mitido que muchas aplicaciones subnacionales del IDH se hayan calculado con datos referidos a los ingresos en reemplazo del PIB per cápita. ${ }^{10}$

De acuerdo con el tratamiento aplicado a las variables de salud y educación, cabría en esta instancia analizar la correlación del indicador de ingreso con el utilizado por el PNUD. Sin embargo, dicho análisis carece de sentido ya que no aportaría información acerca de la correspondencia entre la nueva variable y la capacidad a evaluar, dada la escasa validez del indicador original.

En cuanto al segundo aspecto a considerar, la forma en que se distribuye la renta en los territorios, la realización de ajustes una vez calculado el índice se torna compleja por la imposibilidad de obtener datos actualizados para construir coeficientes en todos los países y, además, con tal procedimiento se invalida el ordenamiento obtenido a partir del cálculo de la medida sin la corrección distribucional. Estas consideraciones justifican la opción por la segunda alternativa metodológica.

De acuerdo con el concepto de titularidades (entitlements) propuesto por Amartya Sen $(1971,1987 a, 1987 b, 1988)$ para diferenciar las capacidades de las personas de su expresión en resultados económicos, se abren dos posibles alternativas para incorporar en la estructura del IDHT la cuestión de la distribución de la renta; agregar algún indicador de incidencia de la pobreza, o bien uno que refleje las dificultades de acceso al mercado laboral. Una determinada unidad geográfica puede arrojar un nivel de ingreso per cápita relativamente alto, pero a la vez presentar un significativo porcentaje de población en situación de pobreza o elevadas tasas de desempleo y/o subocupación, reflejando con ello una alta concentración de la renta en determinados grupos. El problema no sería tanto la escasez de recursos materiales como la incapacidad por parte de muchas personas de acceder efectivamente a ellos. La consideración de este tipo de variables en la medición aumentaría entonces la correspondencia con el concepto original.

Los indicadores de pobreza suponen cierto grado de complejidad en su cálculo, lo que hace que en cada país se utilicen diferentes parámetros metodológicos, disminuyendo el valor comparativo de los datos. De ahí que la alternativa escogida consistiera en incorporar a la tercera dimensión del IDHT un indicador de dificultades laborales que agrega el porcentaje de la población económicamente activa (PEA) que se encuentra desempleada y el porcentaje de la PEA en situación de subocupación horaria. Estas variables reflejan la magnitud de las deficiencias en el mercado de trabajo y, por tanto, la existencia de una mayor o menor concentración

\footnotetext{
${ }^{10}$ PNUD, 2000, 2004b, 2005; Hanham et al., 2000. Incluso el informe mundial PNUD (2006) presenta un análisis desagregado para un conjunto de países, en el que se utiliza el ingreso per cápita de los hogares en sustitución del PIB per cápita.
} 
de la renta. Los indicadores de empleo completan así la información arrojada por el ingreso al representar el modo en que las personas materializan sus capacidades.

En resumen, el tercer componente del IDHT quedaría conformado por el ingreso per cápita de los hogares, para medir la disponibilidad de renta, y el indicador de dificultades laborales, expresado como el porcentaje de la PEA que no está desocupada ni en situación de subocupación horaria, para evaluar la forma en que el ingreso se distribuye en cada unidad espacial.

\section{Composición y metodología de elaboración del IDHT}

Como resultado de los análisis realizados, el IDHT quedaría conformado como aparece en el cuadro 1.

\section{Cuadro 1 \\ Composición del índice de desarrollo humano territorial}

\begin{tabular}{ll}
\hline Dimensiones & \multicolumn{1}{c}{ Indicadores } \\
\hline Salud & Tasa de sobrevivencia en menores de cinco años \\
Educación & Tasa bruta de matriculación combinada primaria y secundaria \\
& Nivel educativo de personas de 25 años y más \\
Nivel de vida & $\begin{array}{l}\text { Ingreso per cápita de los hogares } \\
\text { Porcentaje de la PEA que no se encuentra desocupada ni en situación } \\
\text { de subocupación horaria }\end{array}$ \\
\hline
\end{tabular}

Fuente: Elaboración propia.

El método de cálculo del IDHT sigue el procedimiento diseñado por el PNUD. El objetivo consiste en medir el progreso medio de las unidades espaciales en cada país, procurando captar las brechas que entre ellas se presentan en términos de logros en salud, educación y nivel de vida. Antes de calcular el índice sintético, es necesario crear un subíndice para cada uno de sus componentes. Para realizar este procedimiento se adoptó el mismo criterio de normalización del IDH-PNUD, cuyo resultado es la diferencia, expresada en términos de proporción, entre los valores máximo y mínimo.

En el caso del IDHT la normalización de la tasa de sobrevivencia en menores de cinco ańos se realiza considerando como valores máximo y mínimo las tasas de tres por mil y 130 por mil, según surge del examen de los datos correspondientes a los países de la región para el año 2001 (PNUd, 2003: 263). El cálculo del indicador de ingresos toma como valores de referencia los estipulados por el PNUD (40,000 y 100 dólares).

\footnotetext{
${ }^{11} \mathrm{El}$ ingreso per cápita de los hogares se normaliza a partir de los logaritmos, sobre el supuesto de que lograr un nivel digno de desarrollo humano no exige ingresos ilimitados.
} 
Para el resto de las variables, al tratarse de porcentajes, se consideran como valores máximo y mínimo 100 y 0 , respectivamente.

\section{Cuadro 2}

\section{Valores normativos de referencia para el cálculo del IDHT}

\begin{tabular}{lrr}
\hline \multicolumn{1}{c}{ Indicador } & Valor máximo & Valor mínimo \\
\hline Tasa de mortalidad en menores de cinco años & & \\
(tasa de sobrevivencia = 1-TM en menores de cinco años) & $130 \times 1,000$ & $3 \times 1,000$ \\
$\begin{array}{l}\text { Tasa bruta de matrícula combinada primaria y } \\
\text { secundaria (\%) }\end{array}$ & 100 & 0 \\
$\begin{array}{l}\text { Nivel educativo de la población de 25 años y más (\%) } \\
\text { Ingreso per cápita de los hogares (PPC en dólares) }\end{array}$ & 100 & 0 \\
$\begin{array}{l}\text { PEA que no se encuentra desocupada ni en situación } \\
\text { de subocupación horaria (\%) }\end{array}$ & 40,000 & 100 \\
\hline
\end{tabular}

Fuente: PNUD (2003: 263).

En seguida se construyen los índices para cada una de las dimensiones. El desempeño se expresa como un valor entre 0 y 1 aplicando la siguiente fórmula general.

$$
\text { Índice del componente }\left(I_{i j}\right)=\frac{\text { valor real - valor mínimo }}{\text { valor máximo - valor minimo }}
$$

Luego se combinan los valores para crear el índice por componente de acuerdo con las siguientes ponderaciones

\begin{tabular}{ll}
\hline Salud & Tasa de sobrevivencia en menores de cinco ańos \\
Educación & 1/3 Tasa de matrícula combinada primaria y secundaria \\
& 2/3 Nivel educativo de personas de 25 ańos y más \\
Nivel de vida & 1/3 Ingreso per cápita de los hogares \\
& 2/3 Indicador laboral \\
\hline
\end{tabular}

La asignación de las ponderaciones se corresponde, en el componente de educación, con el peso otorgado en el IDH-PNUD a la tasa de matrícula combinada $(1 / 3)$ y a la tasa de alfabetización de adultos $(2 / 3)$, reemplazada por el nivel educativo de personas de 25 años y más. En el aspecto nivel de vida, se otorgó una mayor ponderación (2/3) al indicador de dificultades laborales, esto responde, por un lado, a la premisa de que las condiciones laborales actuarían como factores condicionantes del nivel de ingresos alcanzado por las personas y, por otro, a que los indicadores del mercado laboral reflejan, a diferencia del ingreso, una materia-

\footnotetext{
${ }^{12}$ Según Banco Mundial (2005).
} 
lización de las capacidades, tener empleo constituye un objetivo deseable por sí mismo.

Una vez calculados los subíndices, la determinación del IDHT se realiza a través de un promedio simple de los tres componentes. ${ }^{13} \mathrm{El}$ valor de la medida oscila entre 0 y 1 , valores cercanos a 0 indican menores niveles relativos de desarrollo humano.

\section{El IDHT: los casos de Argentina y México}

Con el objetivo de validar el método de cálculo del IDHT, en este apartado se describen sintéticamente los resultados de su aplicación en Argentina y México. La elección de estos países responde a la disponibilidad de información estadística; pero también al hecho de que el nuevo índice ha sido formulado para evaluar, de manera específica, las disparidades territoriales en las naciones que han alcanzado un nivel de logros relativamente elevado.

El análisis parte del cálculo del IDHT para cada una de las unidades subnacionales, provincias argentinas y entidades federativas mexicanas. Se compara el comportamiento de los nuevos indicadores con el de las variables originales utilizadas en la construcción del IDH-PNUD, se estudian las diferencias en cuanto a los ordenamientos territoriales generados y se analiza la sensibilidad territorial de cada uno de los indicadores. Luego se derivan algunas conclusiones relativas a la capacidad de la nueva medida para evaluar las distancias que separan a las entidades espaciales de uno y otro país.

\subsection{El desarrollo humano en las provincias argentinas}

El PNud presenta en el informe 2005 el cálculo del IDH y sus componentes para las 24 provincias en las que se organiza el territorio nacional, la medición considera como base el año 2004. Para fines de comparación, el ejercicio aquí realizado toma como referencia esos parámetros.

\subsubsection{Análisis de las dimensiones que lo componen}

En las gráficas I y II se muestra la clasificación de las provincias en la dimensión salud según IDH-PNUD e IDHT.

\footnotetext{
${ }^{13}$ El tema de la ponderación ha sido ampliamente debatido por autores como Kelley (1991), Das (1993), Ravallion (1997) o López y Vélez (2003), entre otros. Los impulsores del IDH argumentan que las tres dimensiones tienen importancia similar para el desarrollo humano, además de que están muy interrelacionadas (Ul Haq, 2003: 128).
} 


\section{Gráfica I}

\section{Clasificación de las provincias según índice de salud, IDH-PNUD. Argentina, 2004}

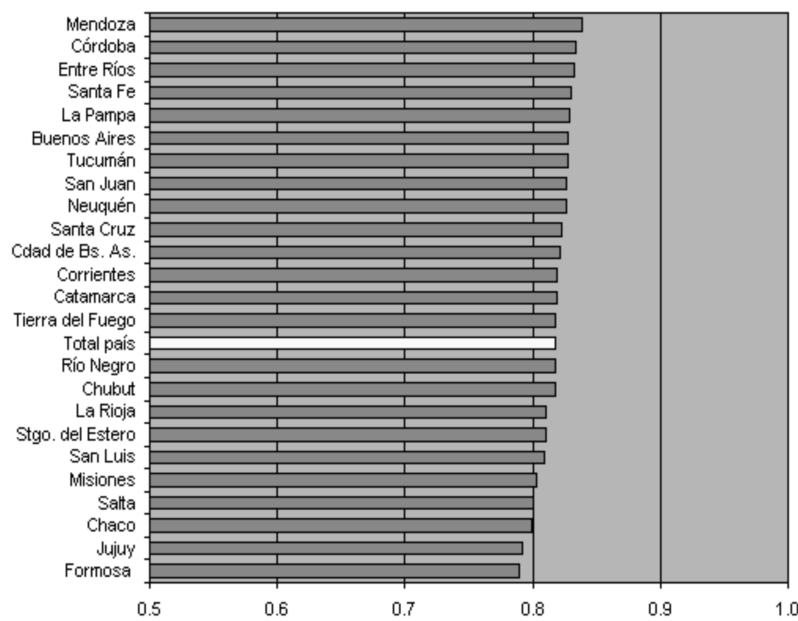

Fuente: Elaboración propia con base en datos del PNUD (2005).

\section{Gráfica II}

\section{Clasificación de las provincias según índice de salud, IDHT.} Argentina, 2004

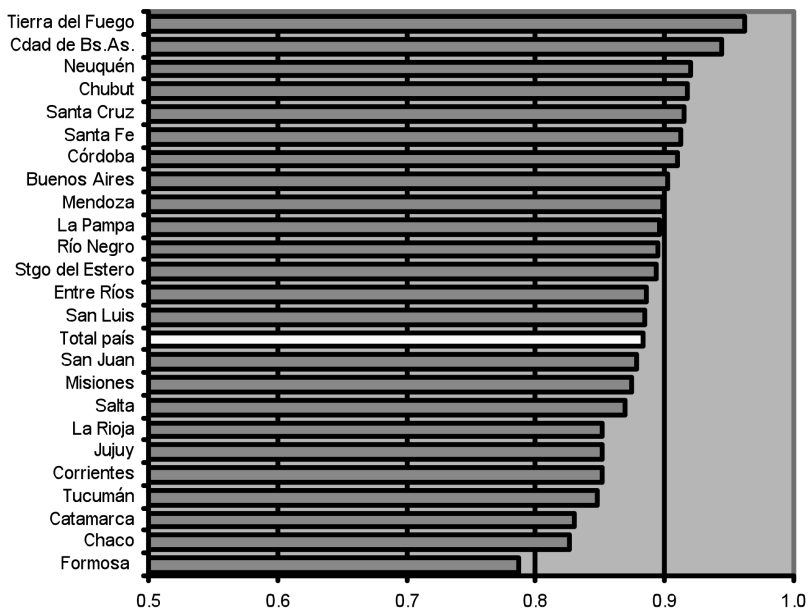

Fuente: Elaboración propia con base en datos del INDEC (2004) y de la Dirección de Estadísticas e Información en Salud (2005). 
La lectura comparada permite derivar dos conclusiones generales: la relativa mayor homogeneidad con que se distribuye la esperanza de vida al nacer respecto de la tasa de supervivencia infantil y el ordenamiento que, de las jurisdicciones provinciales, genera una y otra clasificación.

Según el cálculo del componente de salud medido a partir de la metodología del IDH-PNUD, la provincia que registra la situación más favorable es Mendoza, acompañada en los primeros puestos por un conjunto de jurisdicciones del centro nacional. Los lugares intermedios los ocupan provincias que pertenecen indistintamente a las regiones sur, norte y centro-oeste del país. Mientras que en los últimos puestos se posicionan cinco jurisdicciones del norte, siendo Formosa la entidad más rezagada.

Como lo evidencian diferentes estudios que analizan el perfil de desarrollo regional de la República Argentina (Rofman y Romero, 1998; Vaca, 2004; PNUD, 2005, entre otros), las provincias norteńas constituyen, en términos de indicadores de pobreza, cobertura de servicios básicos, condiciones laborales, participación en el PIB, etc., la región más desfavorecida. Mientras que las jurisdicciones del sur junto con la Ciudad de Buenos Aires presentan, en términos relativos, los indicadores más favorables.

La medición del componente sanitario de acuerdo con el método del IDHT, sitúa a Tierra del Fuego en el primer puesto de la clasificación, y nuevamente aparece Formosa como la provincia más rezagada. La distribución generada a partir de la tasa de sobrevivencia en menores de cinco años, da mejor cuenta de la regionalización mencionada, al colocar a las jurisdicciones patagónicas junto con la capital nacional, en los primeros lugares y en el extremo inferior a las provincias norteńas. Resultados que estarían avalando la mayor adecuación del nuevo indicador para explicar las diferencias que, en términos de salud, distinguen a las provincias argentinas.

El análisis de variabilidad territorial aplicado a las series de datos y fundamentado en el cálculo de la desviación estándar, valores extremos y rangos, indica la mayor sensibilidad de la nueva variable para reflejar los logros sanitarios en cada una de las jurisdicciones.

La dispersión de los datos refleja la mayor heterogeneidad con que se distribuye el índice de salud según su cálculo a partir del IDHT, pero también indica que el complemento de la tasa de mortalidad en menores de cinco años es un indicador escasamente exigente a los desafíos que un país con el perfil de desarrollo de Argentina está en condiciones de imponerse. La mayoría de las jurisdicciones adopta valores muy cercanos al considerado óptimo para la dimensión.

En conclusión, el aspecto salud, medido a partir de la tasa de sobrevivencia en menores de cinco años, resulta una alternativa metodológica más pertinente para evaluar el estado sanitario que diferencia a las provincias argentinas. En términos comparativos con la variable original, 


\section{Gráfica III}

Dispersión de los valores provinciales, índices de salud.

Argentina, 2004

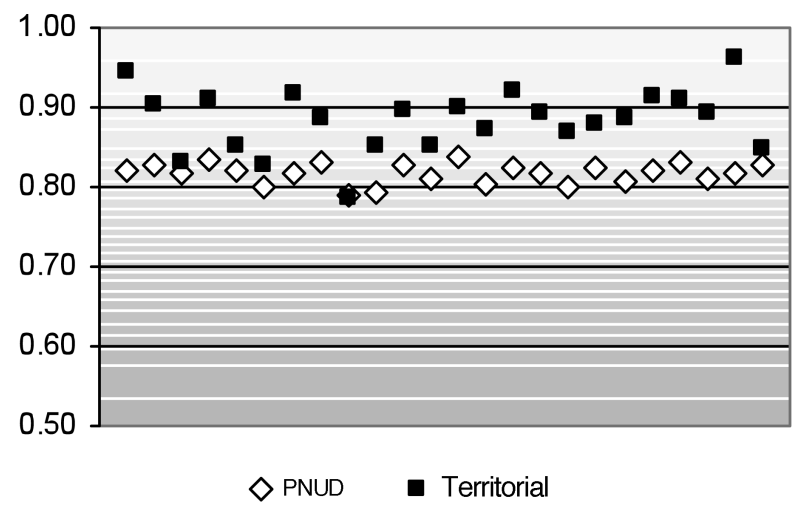

Fuente: Elaboración propia con base en fuentes estadísticas oficiales.

\section{Gráfica IV}

Clasificación de las provincias según índice de educación, IDH-PNUD. Argentina, 2004

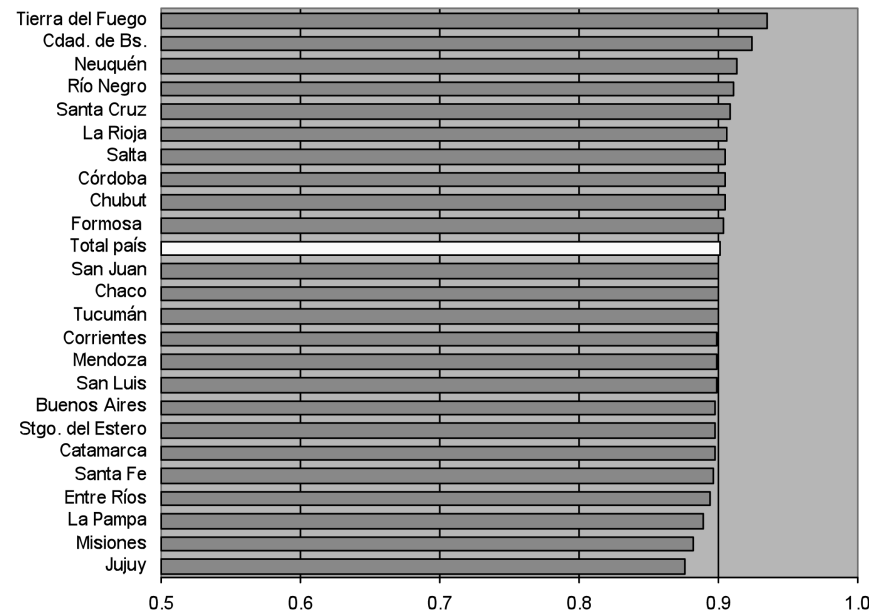

Fuente: Elaboración propia con base en datos del PNUD (2005). 
Gráfica V

Clasificación de las provincias según índice de educación, IDHT. Argentina, 2004

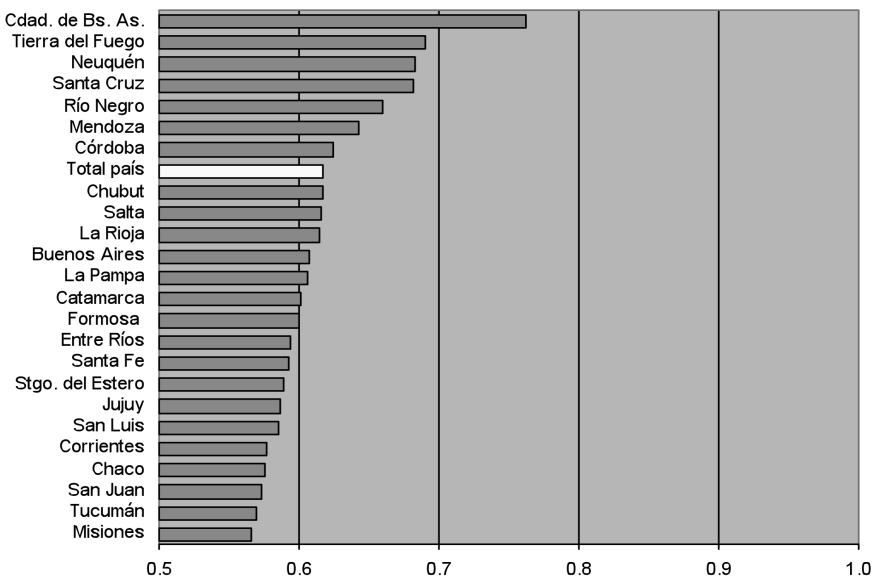

Fuente: Elaboración propia con base en datos del INDEC-EPH (2004) y Red Federal de Información Educativa (2006).

presenta una mayor heterogeneidad en su distribución y arroja una clasificación de las unidades administrativas más acorde con la regionalización del desarrollo. Hay que señalar, sin embargo, que el nuevo indicador adopta valores muy cercanos al óptimo, que lo posiciona como una medida escasamente exigente para reflejar los logros en salud de una determinada población. El mismo análisis se aplicó en el tema educación.

La primera conclusión que suscita la observación de las gráficas, es la significativa mayor heterogeneidad con que se distribuye el componente educativo medido según el IDHT, así como la adopción de valores sensiblemente inferiores a los registrados en la dimensión original. En lo referente al ordenamiento de las provincias, se producen importantes modificaciones al pasar de una clasificación a otra, si bien en ambas series se mantiene, en términos generales, la regionalización descrita en el análisis del aspecto sanitario. Las provincias del sur, junto con la Ciudad de Buenos Aires, se ubican en los primeros puestos y las jurisdicciones norteñas en los últimos.

La incorporación del indicador de nivel de estudios supone una importante modificación en la medición de los logros educativos. La mayor exigencia de la nueva variable hace que prácticamente todas las provincias cambien su posición en el ordenamiento nacional y que los valores adoptados sean muy inferiores a los arrojados por la dimensión original.

Estas argumentaciones se refuerzan a partir del análisis de las medidas de dispersión para cada serie de datos, que ponen de manifiesto la mayor 


\section{Gráfica VI \\ Dispersión de los valores provinciales, índices de educación. Argentina, 2004}

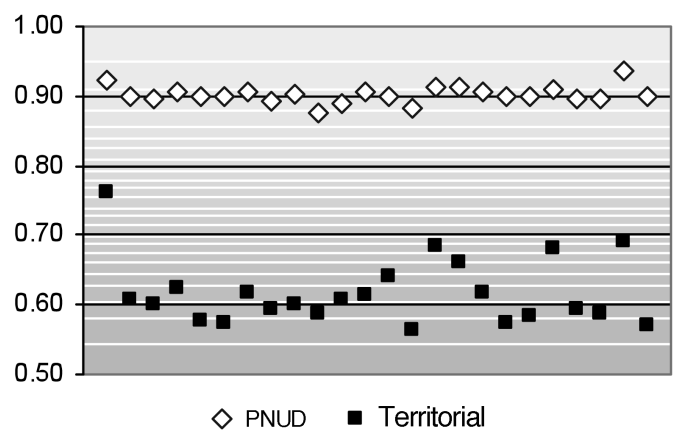

Fuente: Elaboración propia con base en fuentes estadísticas oficiales.

capacidad del componente educativo del IDHT para reflejar las disparidades existentes entre las provincias.

La dimensión educativa medida según el IDH-PNUD se distribuye entre las provincias de manera claramente homogénea y en torno a valores considerados óptimos. Asimismo se refleja la mayor dispersión del componente educativo del IDHT siendo, además, una medida más exigente al colocarse todos los valores muy por debajo de la situación considerada óptima.

En resumen, el componente educativo evaluado según el IDHT recoge de manera más precisa las diferencias en países relativamente avanzados en términos de desarrollo humano. La nueva medida arroja una distribución mucho más heterogénea que el índice original y, al incorporar un indicador más exigente, hace que los territorios adopten valores inferiores a los registrados en la misma dimensión del IDH-PNUD, lo que estaría señalando mayor rigor para medir la distancia que aún tienen por recorrer los territorios subnacionales en materia de oportunidades educativas.

El cálculo del componente nivel de vida arroja las siguientes clasificaciones.

Las gráficas viI y viII muestran los valores menores que adopta el componente medido según la metodología del PNUD. En cuanto a la forma en que se distribuyen los logros, no se aprecia a simple vista una mayor o menor homogeneidad en alguna de las series.

Los reposicionamientos generados a partir de la nueva metodología se interpretan como el resultado de la incorporación del indicador de dificultades laborales, dado que el PNUD (2005) utilizó para el cálculo del componente original el ingreso per cápita de los hogares en reemplazo del PIB per cápita. 


\section{Gráfica VII}

Clasificación de las provincias según índice de nivel de vida, IDH-PNUD. Argentina, 2004

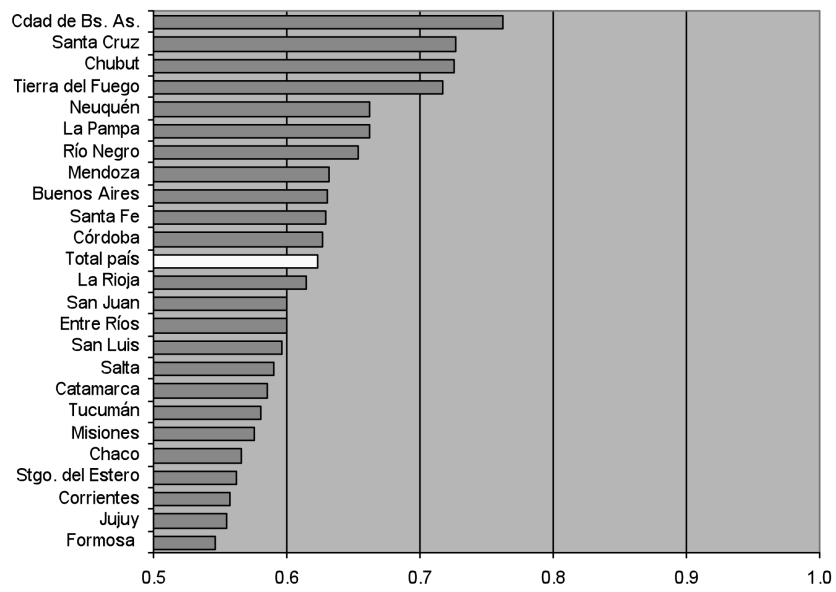

Fuente: Elaboración propia con base en datos del PNUd (2005).

\section{Gráfica VIII}

Clasificación de las provincias según índice de nivel de vida, IDHT. Argentina, 2004

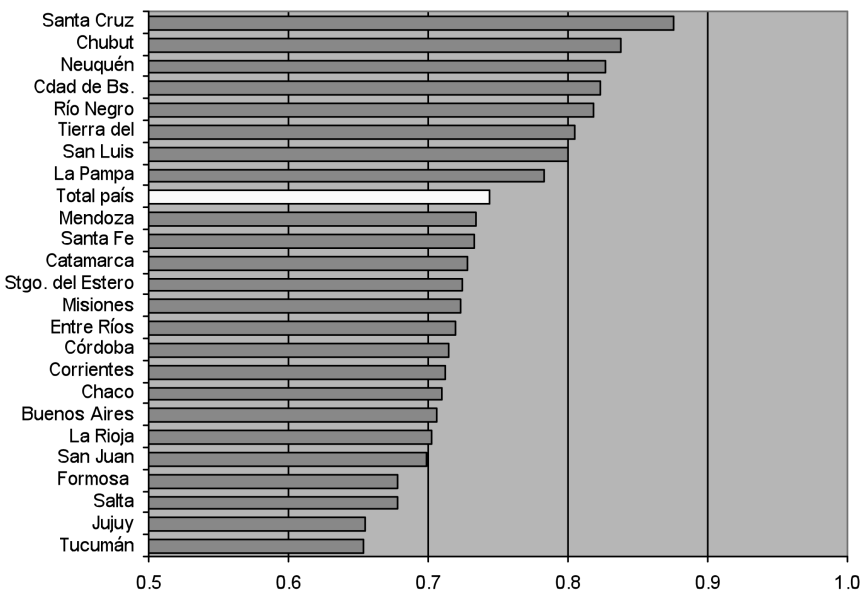

Fuente: Elaboración propia con base en datos del INDEC-EPH (2004). 


\section{Gráfica IX \\ Dispersión de los valores provinciales, índices de nivel de vida. Argentina, 2004}

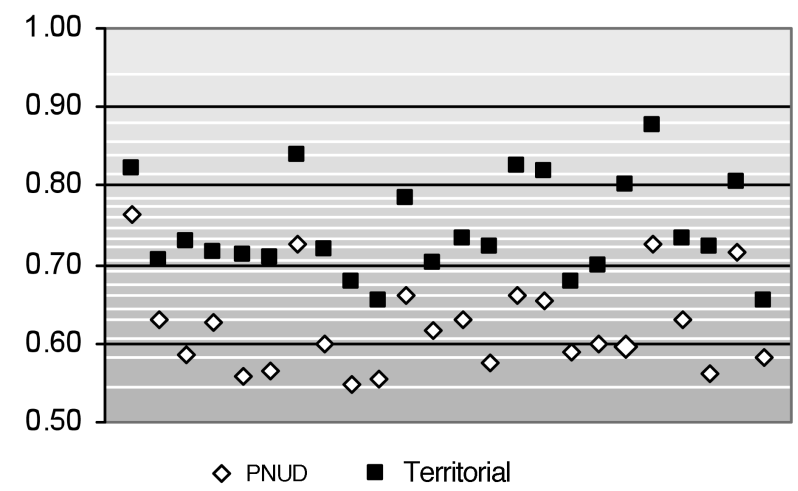

Fuente: Elaboración propia con base en fuentes estadísticas oficiales.

Ambas series reflejan la distribución regional del desarrollo en el país, donde las provincias patagónicas y la capital nacional se ubican en las primeras posiciones, si bien la Ciudad de Buenos Aires se posiciona según la serie original en el primer puesto, aunque luego pasa al cuarto lugar. Es de destacar que ninguna de las provincias norteñas se coloca por encima de la media nacional.

Las medidas de variabilidad que se describen en la serie de datos de la dimensión según el IDHT, presentan valores levemente superiores a los arrojados en el IDH-PNUD.

La gráfica IX aporta evidencia acerca de la desigual distribución del componente evaluado a partir de ambas metodologías. Destaca que la dimensión medida según el IDHT presenta valores superiores a los del IDH-PNUD, entre 0.8761 y 0.6538 el primero, y entre 0.7622 y 0.5463 el segundo. El indicador de dificultades laborales empuja, por tanto, hacia arriba los valores alcanzados por las provincias.

En síntesis, el aspecto nivel de vida calculado con el nuevo procedimiento, presenta una sensibilidad territorial un poco superior al mismo componente del IDH-PNUD. Los efectos de la corrección de distribución se hacen notar en los reposicionamientos de las provincias en la clasificación y en el aumento de los valores alcanzados por las mismas en el componente. Los cambios en el ordenamiento reflejan de manera más precisa la distribución de las capacidades de acceso a recursos materiales entre las diversas jurisdicciones subnacionales, de ahí su ventaja frente al procedimiento de medición aplicado por el PNUD para evaluar el componente. 


\subsubsection{Análisis comparado entre indices agregados}

Una vez que se evaluó el comportamiento de los indicadores que componen el IDHT, cabe estudiar el efecto que, en términos comparativos con el IDH-PNUD, produce el nuevo índice agregado. La clasificación general de las provincias argentinas según IDH-PNUD e IDHT se configura como se muestra en las siguientes gráficas.

\section{Gráfica X}

\section{Clasificación de las provincias según IDH-PNUD. Argentina, 2004}

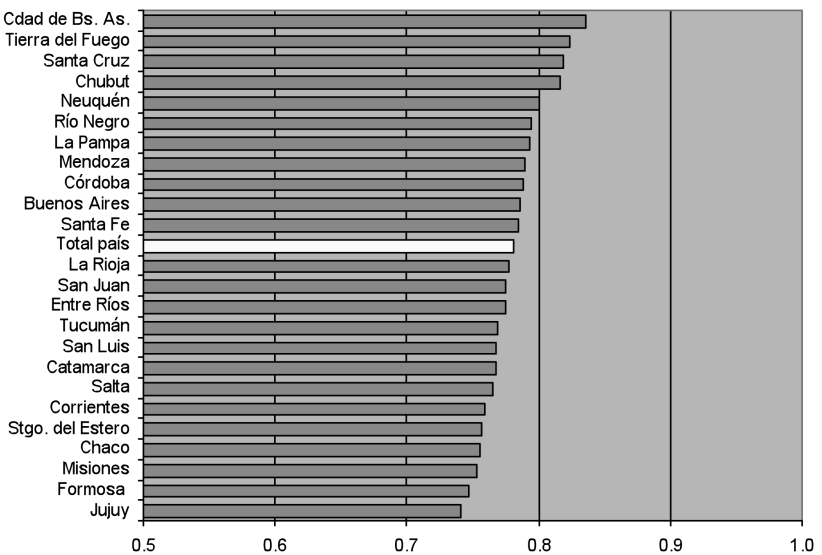

Fuente: Elaboración propia con base en datos del PNUD (2005).

\section{Gráfica XI}

\section{Clasificación de las provincias según IDHT. Argentina, 2004}

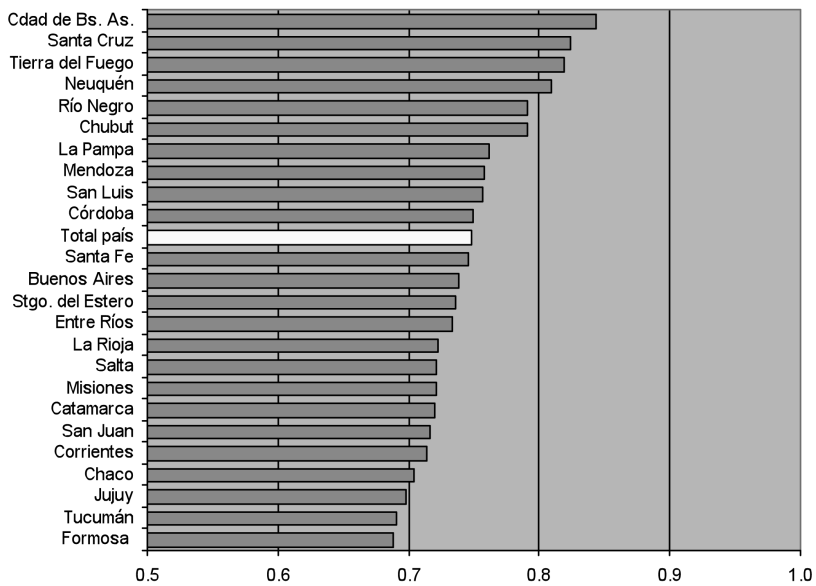

Fuente: Elaboración propia con base en fuentes estadísticas oficiales. 


\section{Gráfica XII \\ Dispersión de los valores provinciales, IDH-PNUD e IDHT. Argentina, 2004}

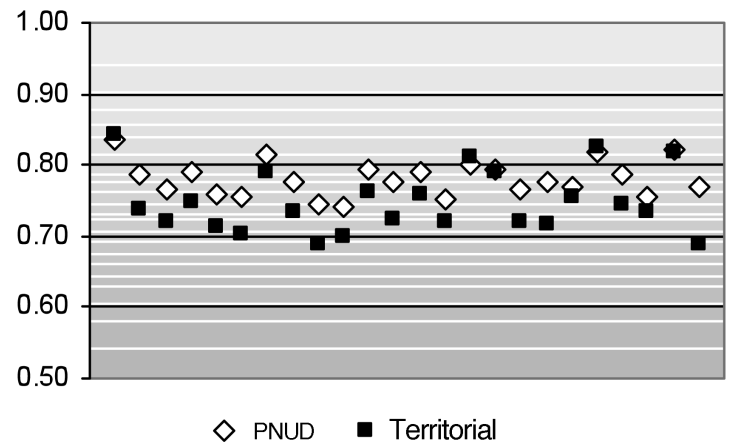

Fuente: Elaboración propia con base en fuentes estadísticas oficiales.

Si bien en la clasificación del IDH-PNUD existe una escala gradual que marca cierta diferencia entre una provincia y otra, los valores aparecen distribuidos de manera relativamente homogénea, la mayoría de las jurisdicciones se ubica en la franja que va de 0.70 a 0.80 .

Tanto la desviación estándar como la amplitud del rango de las series de datos correspondientes a cada índice, muestran la mayor heterogeneidad con que se distribuyen los logros en desarrollo humano medidos según la nueva metodología. De lo que se infiere la mayor capacidad del IDHT para reflejar la realidad del desarrollo en un país como Argentina.

Aunque ambas series se distribuyen de manera similar, los valores alcanzados por las provincias en el IDHT presentan mayor heterogeneidad. En la serie configurada por el nuevo índice, las jurisdicciones provinciales adoptan valores comprendidos en una franja algo más amplia que en el caso del IDH-PNUD.

En síntesis, los resultados de la aplicación del IDHT en las provincias argentinas, teniendo en cuenta que se trata de un país con un perfil de desarrollo que lo coloca en el primer puesto de la clasificación del PNUD para las naciones de América Latina, permiten concluir acerca de la mayor validez de la nueva medida para aproximar la forma en que se distribuyen los logros en desarrollo humano. Los cambios de posición que suscita el IDHT, en términos comparativos con el IDH-PNUD, reflejan la distribución territorial del desarrollo evidenciada en diferentes investigaciones. Las provincias del sur junto con la Ciudad de Buenos Aires arrojan los índices más elevados, mientras que las del norte presentan los mayores rezagos. 


\subsection{El desarrollo humano en los estados mexicanos}

Para estudiar el comportamiento del IDHT en las 32 entidades federativas mexicanas se tomaron como referencia las series de datos estadísticos correspondientes al año 2003, dado que el último cálculo del IDH-PNUD desagregado a escala estadual fue publicado por el PNUD (2004b) y corresponde a dicho ańo.

\subsubsection{Análisis de los indicadores que lo componen}

La medición de los logros en el indicador salud de acuerdo con el IDHPNUD y el IDHT ordena las entidades federativas mexicanas como se muestra las gráfica XIII y XIV.

$\mathrm{Al}$ igual que se observó para el caso de las provincias argentinas, lo que surge de las clasificaciones generadas es la relativa mayor homogeneidad con que se distribuye el componente sanitario entre los estados mexicanos medido según la metodología del PNUD.

México también presenta importantes desequilibrios en la distribución de los indicadores de desarrollo humano, en este caso, el noreste y el noroeste muestran los mayores logros y el centro-oeste y sur del país constituyen las áreas económica y socialmente más rezagadas (OCDE, 2002; PNUD, 2004b). En especial los estados del sur conforman una región con índices cuantitativa y cualitativamente mayores de pobreza, mortalidad infantil y analfabetismo (Dávila et al., 2002: 5).

Aunque con algunas modificaciones en el posicionamiento de los estados, ambas series ubican en los primeros puestos y junto con la capital nacional, a las entidades del norte del país. En contraste, los estados del sur se colocan hacia el final de la serie. Es de destacar que Oaxaca y Chiapas aparecen en las dos clasificaciones como las jurisdicciones más desfavorecidas en materia sanitaria.

En cuanto a las medidas de variabilidad, éstas sugieren que la tasa de sobrevivencia en menores de cinco años se distribuye de manera más heterogénea que la esperanza de vida al nacer, lo que refleja la mayor pertinencia de la nueva metodología para evaluar las disparidades en los logros alcanzados por los estados en cuanto a capacidades sanitarias.

Según se observa, ambas series tienen una distribución similar, sin embargo, la amplitud del rango es mayor en los valores arrojados por la dimensión calculada según el IDHT.

Cabe destacar que, a pesar de las diferencias en niveles de desarrollo que tradicionalmente han distinguido a Argentina de México, éste último país registraba en el año 2004 una esperanza de vida al nacer para el total nacional superior a la de Argentina: 75.3 y 74.6 años, respectivamente. 


\section{Gráfica XIII}

Clasificación de los estados según índice de salud, IDH-PNUD.

México, 2003

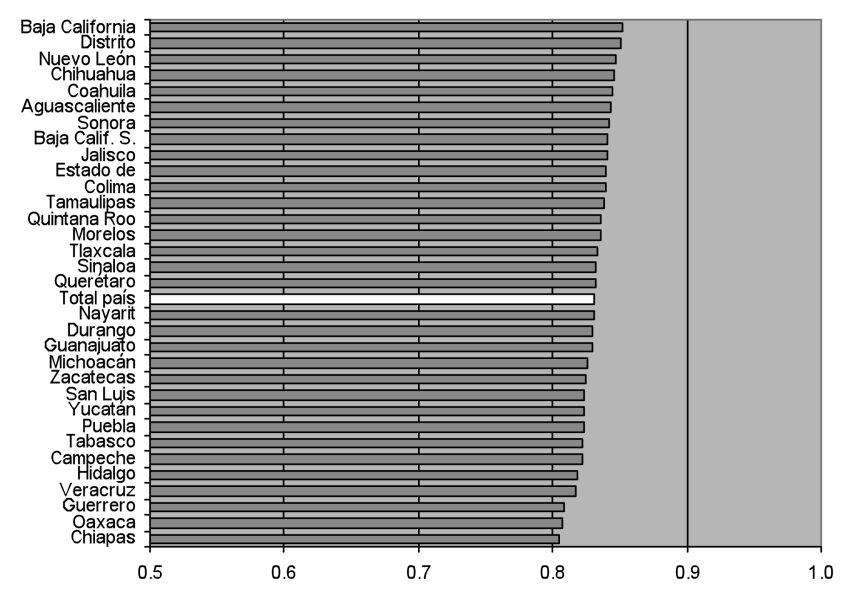

Fuente: Elaboración propia con base en PNUd (2004b).

\section{Gráfica XIV \\ Clasificación de los estados según índice de salud, IDHT. México, 2003}

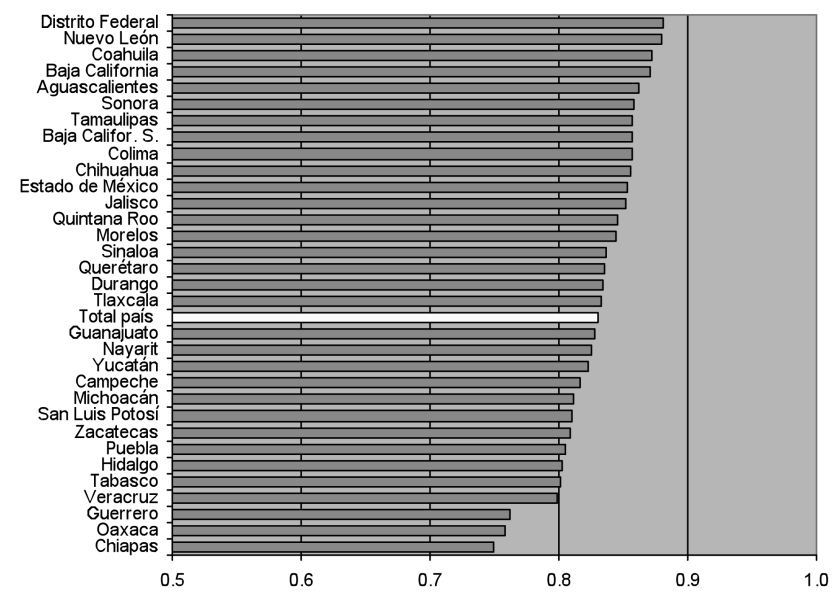

Fuente: Elaboración propia con base en datos del INEGI (2004) y Conapo (2005). 


\section{Gráfica XV}

Dispersión de los valores estatales, índices de salud. México, 2003

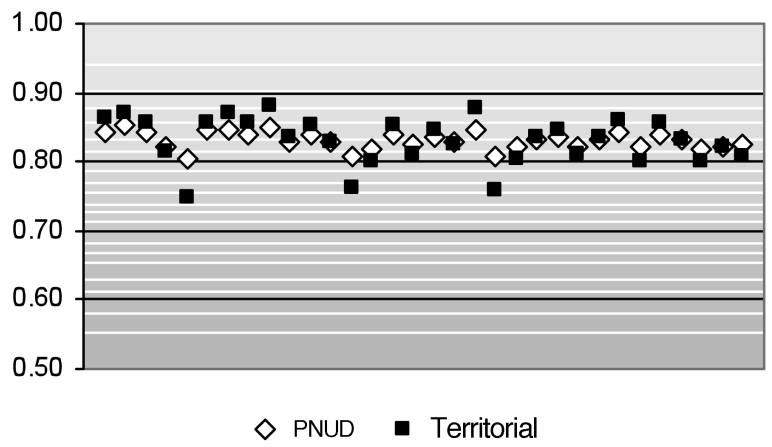

Fuente: Elaboración propia con base en fuentes estadísticas oficiales.

Dado entonces el elevado valor que alcanza la variable original y la relativa homogeneidad con que se distribuye entre los estados, la medición del componente con base en la tasa de sobrevivencia en menores de cinco años resulta más pertinente para observar las disparidades sanitarias en el territorio mexicano. Sin embargo, y al igual que en el caso de Argentina, el nuevo indicador se muestra escasamente exigente al asumir valores próximos a los considerados óptimos.

En el tema de educación se generan las siguientes clasificaciones.

En el caso de los estados mexicanos, también se observa una relativa alta homogeneidad en la distribución del componente educativo medido según el método del PNUD. Para esta dimensión se repite la distribución que coloca en las mejores posiciones a las entidades del norte del país y en las más desfavorecidas a los emplazados en la región sur.

La distribución generada a partir del IDHT permite derivar las mismas conclusiones que para el caso argentino. Por un lado, la mayor heterogeneidad con que se distribuye el componente educativo según la nueva metodología. Por otro, la disminución de los valores alcanzados por las entidades federativas respecto de la dimensión original.

Los resultados del análisis de variabilidad refuerzan esas apreciaciones, los valores de las desviaciones estándar muestran que el aspecto educativo medido con el IDHT alcanza una mayor heterogeneidad en la distribución.

La nueva medida se distribuye de manera más desigual y se muestra como un indicador más exigente. Sin embargo, el índice original también arroja un significativo grado de heterogeneidad, lo que indica que en México aún no se han cumplido los objetivos de equiparación en cuanto al logro de la educación básica. Por tanto, el componente medido sobre 


\section{Gráfica XVI}

Clasificación de los estados según índice de educación, IDH-PNUD. México, 2003

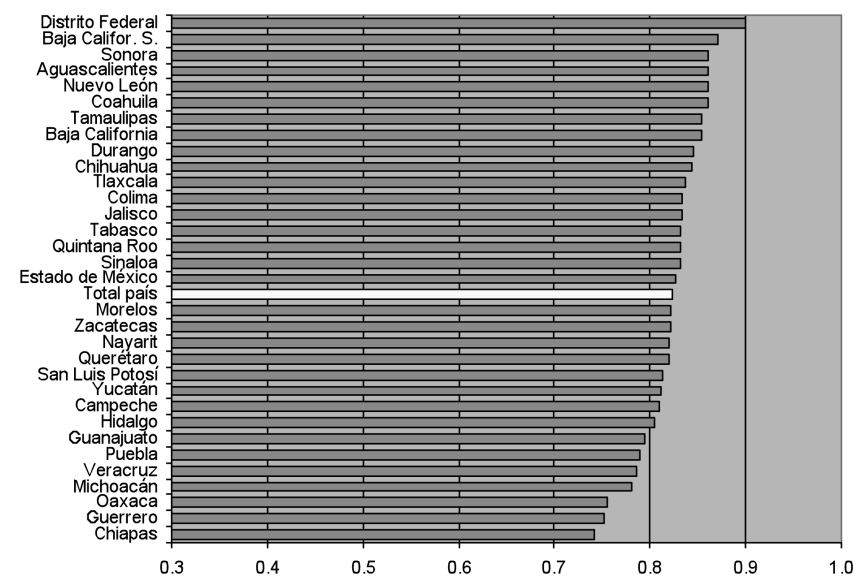

Fuente: Elaboración propia con base en datos del PNUD (2004b).

\section{Gráfica XVII \\ Clasificación de los estados según índice de educación, IDHT. México, 2003}

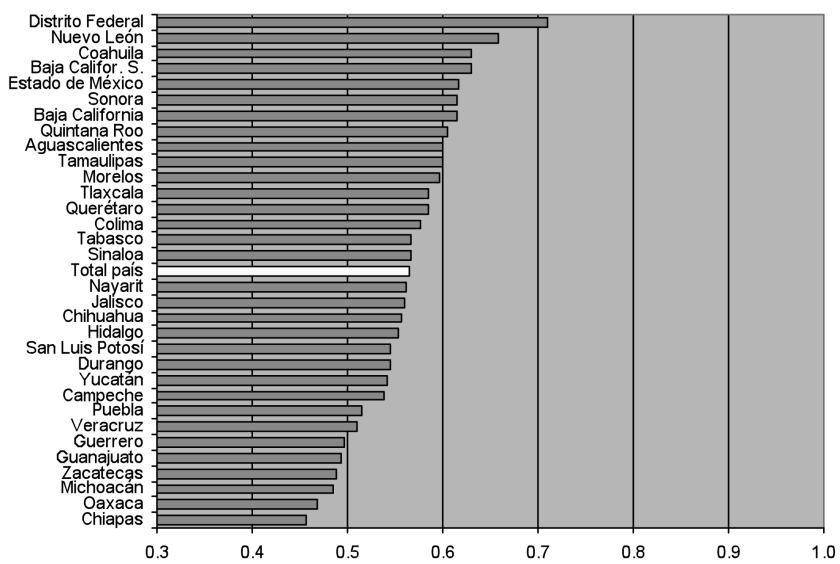

Fuente: Elaboración propia con base en datos de la Dirección Nacional de Planeación, Programación y Presupuesto-SEP (2004). 


\section{Gráfica XVIII}

\section{Dispersión de los valores estatales, índices de educación. México, 2003}

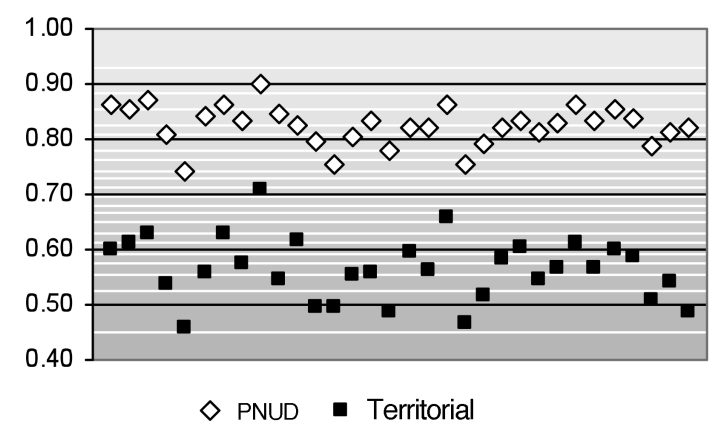

Fuente: Elaboración propia con base en fuentes estadísticas oficiales.

la base de la tasa de alfabetización y la matrícula combinada todavía mantiene una validez significativa.

En conclusión, la comparación del cálculo del componente educativo a partir del IDHT y del IDH-PNUD, muestra que la nueva medida alcanza mayor pertinencia para reflejar las diferencias en logros educativos que separan a las entidades federativas mexicanas. La dimensión evaluada según el IDHT arroja mayor heterogeneidad en su distribución y se muestra más exigente para medir la distancia que tiene por recorrer cada uno de los estados en materia de ampliación de oportunidades educativas.

Los ordenamientos de las entidades federativas mexicanas en el componente nivel de vida según el IDH-PNUD y el IDHT se muestran en las gráficas XIX y Xx.

La distribución según la nueva medida no evidencia diferencias importantes en relación con la clasificación del PNUD, al menos en el sentido de presentar distancias más significativas entre entidades. Sí se observa que la clasificación de los estados mexicanos según la metodología del IDHT genera importantes reordenamientos.

Especialmente en el caso de México, la nueva metodología supone un cambio sustancial en la medición del componente. En primer lugar porque, en el cálculo de la dimensión según IDHT se reemplaza el PIB per cápita por el ingreso per cápita de los hogares. En segundo lugar, porque al incorporar la corrección de distribución mediante el indicador de dificultades laborales, se modifica de manera significativa la situación de cada una de las entidades federativas. Resulta, por tanto, complejo extraer conclusiones comparativas cuando se ha argumentado la escasa validez del PIB per cápita como indicador de la capacidad de acceso a recursos materiales. 


\section{Gráfica XIX}

Clasificación de los estados según índice de nivel de vida, IDH-PNUD. México, 2003

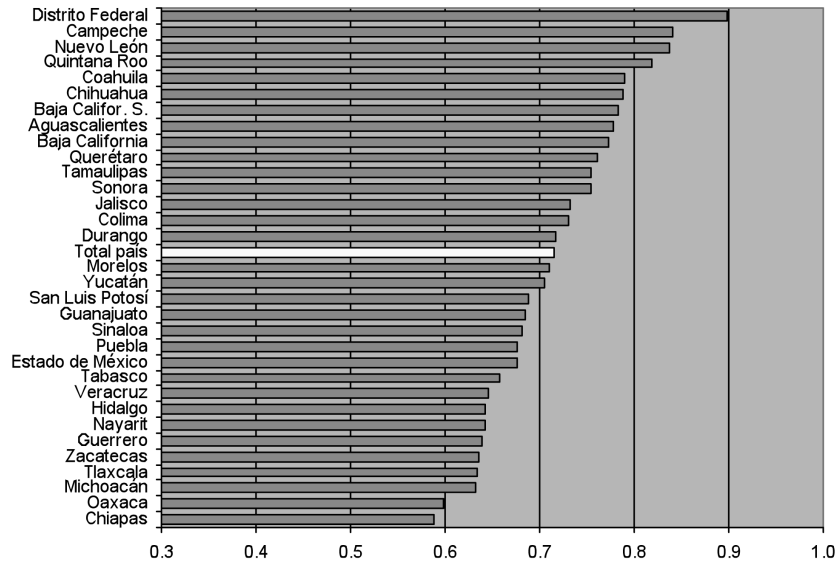

Fuente: Elaboración propia con base en datos del PNUD (2004b).

\section{Gráfica XX}

Clasificación de los estados según índice de nivel de vida, IDHT. México, 2003

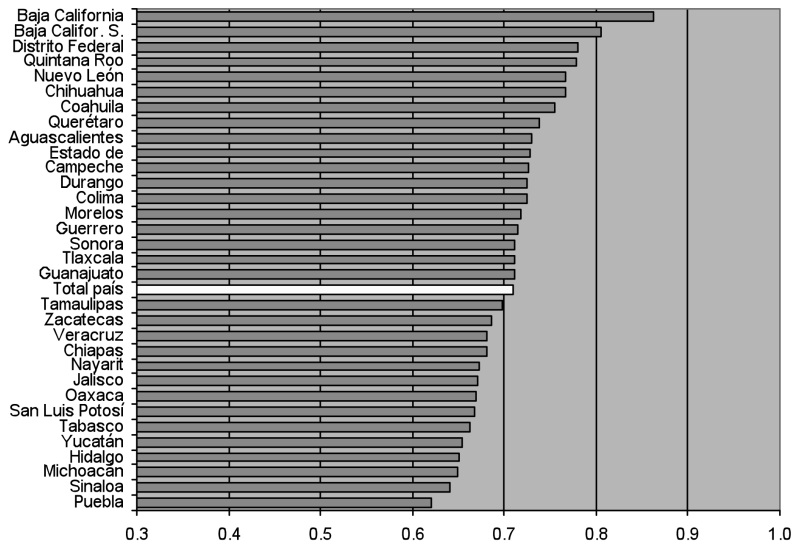

Fuente: Elaboración propia con base en datos de INEGI (2004b). 


\section{Gráfica XXI \\ Dispersión de los valores estatales, índices de nivel de vida. México, 2003}

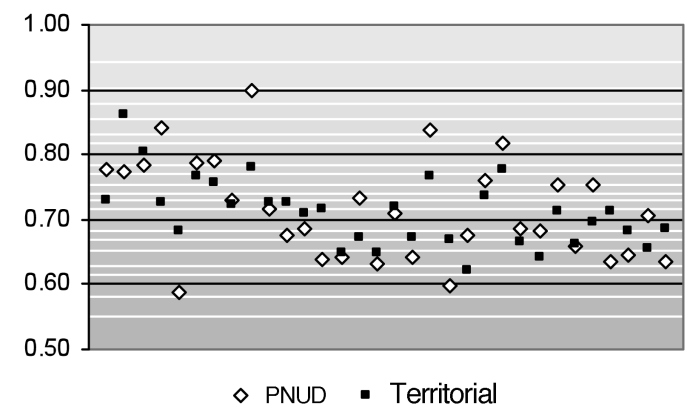

Fuente: Elaboración propia con base en fuentes estadísticas oficiales.

Según las medidas de dispersión, la distribución de la variable original es significativamente más desigual que la que surge de la combinación entre el ingreso per cápita y las tasas de desempleo y subocupación.

En resumen, el componente nivel de vida calculado según el IDHT se distribuye, entre las entidades federativas mexicanas, de manera más homogénea que el mismo componente del IDH-PNUD. Al no poder equiparar el PIB per cápita con el ingreso per cápita de los hogares, no es posible concluir acerca del efecto que produce la incorporación del indicador de dificultades laborales de manera aislada. Sin embargo, de acuerdo con las argumentaciones esgrimidas en apartados anteriores, la clasificación generada por el IDHT estaría evaluando de manera más aproximada las posibilidades de acceso a recursos materiales en el territorio mexicano, dada la mayor validez de los indicadores que incorpora.

\subsubsection{Análisis comparativo entre indices agregados}

El cálculo del IDH-PNUD y del IDHT para las entidades federativas genera las siguientes clasificaciones.

A pesar de las modificaciones en las posiciones ocupadas por los estados, en ambas clasificaciones se mantiene el patrón de distribución territorial del desarrollo. El norte y algunos estados del centro presentan los mayores niveles de logro relativo, mientras la región sur arroja los menores valores. En ambos ordenamientos, el Distrito Federal se ubica en el primer puesto y Chiapas en el último.

$\mathrm{Al}$ analizar los resultados de las medidas de dispersión se observa que el IDHT se distribuye de manera más heterogénea que el IDH-PNUD. Hay que señalar, sin embargo, que la diferencia entre las desviaciones estándar 


\section{Gráfica XXII}

\section{Clasificación de los estados según IDH-PNUD. México, 2003}

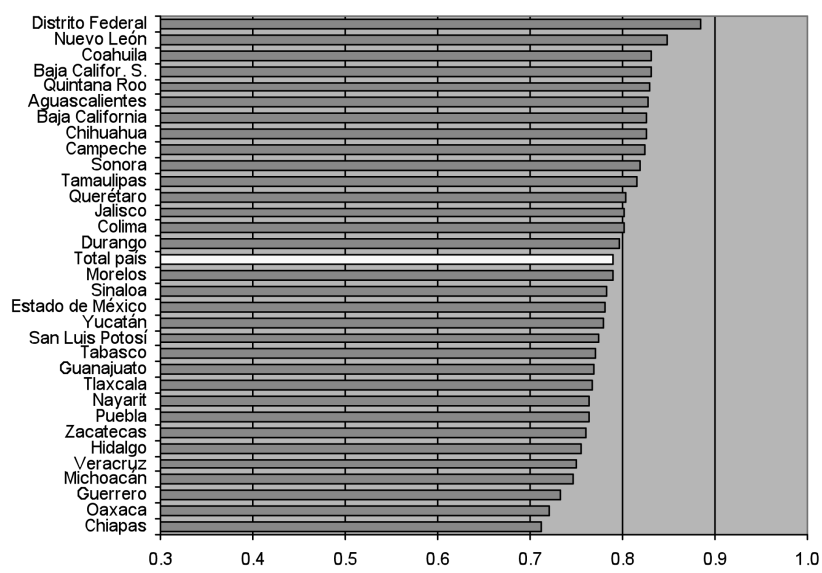

Fuente: Elaboración propia con base en datos del PNUD (2004b).

\section{Gráfica XXIII \\ Clasificación de los estados según IDHT. México, 2003}

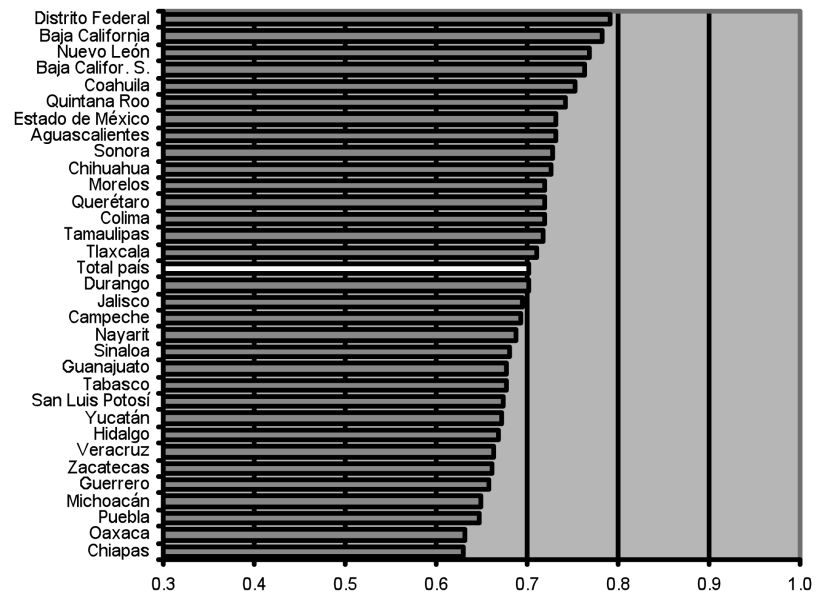

Fuente: Elaboración propia con base en fuentes estadísticas oficiales. 


\section{Gráfica XXIV}

\section{Dispersión de los valores estatales, IDH-PNUD e IDHT. México, 2003}

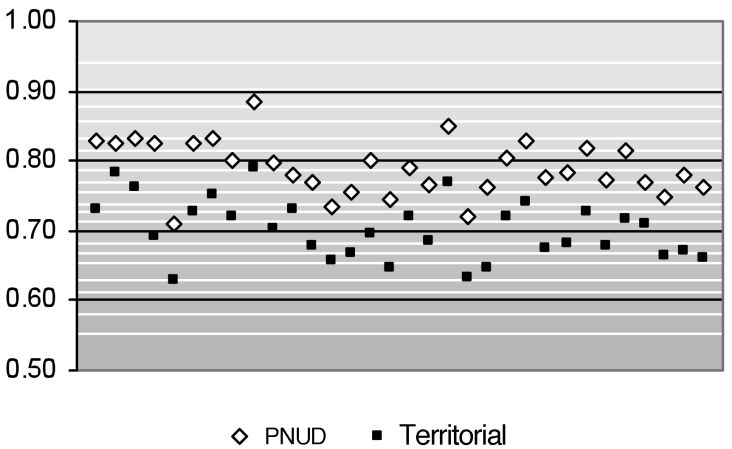

Fuente: Elaboración propia con base en fuentes estadísticas oficiales.

de uno y otro índice es inferior a la observada para las provincias argentinas, dado que la dispersión del IDH-PNUD es significativamente mayor en el caso mexicano. Esta apreciación permite corroborar la hipótesis de la mayor pertinencia del IDHT para evaluar los logros a escala subnacional en países que presentan un mayor grado de avance relativo.

En México, el IDHT se distribuye de manera un poco más desigual y adopta valores sensiblemente inferiores a los que arroja el IDH-PNUD, efecto más pronunciado que en el caso de las provincias argentinas. Ninguna de las entidades mexicanas adquiere en el IDHT valores superiores a 0.80 .

Como conclusión, la comparación entre los valores de los dos índices para los estados de México evidencia la mayor capacidad de la nueva medida para dar cuenta de la forma en que se distribuye el desarrollo humano en el país. Aunque hay que destacar que el IDH-PNUD también presenta, para este caso, un patrón de desarrollo humano significativamente heterogéneo en términos espaciales.

\section{Conclusiones}

La incorporación de la dimensión territorial en el estudio del desarrollo humano aparece como una cuestión de significativa relevancia. En términos de evaluación, el IDH-PNUD tiene la característica de ser una medida flexible y abierta, que se puede modificar en vistas a mejorar su capacidad para aproximar de manera más precisa la realidad del desarrollo en los países y así servir de guía en el diseño, ejecución y evaluación de políticas públicas. 
Una vez constatada la escasa sensibilidad de ese índice para reflejar el desarrollo humano a escala subnacional, y sobre la base de un exhaustivo rastreo y análisis de fuentes de información estadística, fue posible identificar un conjunto de variables susceptibles de ser integradas en una nueva medida con mayor capacidad de discriminación para acotar la desigualdad territorial que presenta el desarrollo humano en los países de América Latina.

En el aspecto salud, la tasa de sobrevivencia en menores de cinco años se muestra más pertinente, por su validez conceptual, posibilidad de cálculo desagregado y sensibilidad territorial, que la esperanza de vida al nacer. En el componente educación se seleccionó, en reemplazo de la alfabetización y para dar cuenta de los conocimientos acumulados, un indicador de nivel educativo en personas de 25 años y más, al que se agregó, para reflejar la capacidad de retención del sistema educativo, la matrícula escolar restringiéndola a los niveles de primaria y secundaria. La permanencia de esta última variable en el índice responde al hecho de haber constatado que se trata de uno de los pocos indicadores disponibles de manera desagregada en todos los países de la región. Finalmente, en lo relativo al tema nivel de vida, el PIB per cápita se sustituyó por el ingreso per cápita, considerado una medida con mayor validez conceptual; se incorporó, además, un indicador de dificultades laborales para aproximarnos a la forma en que se distribuye la renta en cada territorio.

La aplicación del indice de desarrollo humano territorial, elaborado a partir de las variables reseñadas, en Argentina y México sugiere algunas conclusiones vinculadas a la pertinencia de la nueva metodología.

En términos generales, el IDHT resulta una medida más adecuada para reflejar la desigualdad con que se distribuyen las capacidades en los países, especialmente las referidas a salud y educación. En el componente sanitario la tasa de supervivencia en menores de cinco años sigue siendo un indicador poco exigente para los desafíos que países como los analizados están en condiciones de imponerse. En el aspecto educación, la nueva metodología ofrece un panorama más ajustado a la realidad educativa de naciones con niveles de desarrollo relativamente elevados y a las brechas que aún tienen por recorrer los distintos territorios. En cuanto al nivel de vida, los resultados no parecen tan concluyentes, la nueva medida más que presentar una mayor sensibilidad territorial genera importantes reposicionamientos que reflejan, de manera más precisa, la forma que adquiere la distribución de las capacidades vinculadas al acceso a recursos materiales por parte de una determinada población.

Por último, a pesar de las coincidencias observadas en los dos casos analizados, hay que destacar que el IDH-PNUD y sus componentes se distribuyen de manera mucho más heterogénea entre las entidades federati- 
vas mexicanas que entre las provincias argentinas. Lo que estaría señalando que la metodología original aún resulta relativamente pertinente para evaluar los logros en desarrollo humano en países que, como México, no han alcanzado a cubrir, de manera territorialmente equilibrada, algunas de las capacidades más elementales.

En síntesis, la elaboración y aplicación empírica del IDHT han aportado elementos que fundamentan la importancia de la dimensión territorial en el análisis del desarrollo humano. Al igual que el IDH-PNUD, se trata de una metodología abierta susceptible de modificaciones para mejorar su validez. Estos cambios se deben encaminar a fundamentar la formulación de indicadores que midan, junto con los aspectos individuales evaluados por el IDH, las dimensiones colectivas espacialmente localizadas, aquéllas de las que, en cada territorio, disponen las personas para adquirir las capacidades y titularidades que posibilitan alcanzar una vida valiosa.

\section{Bibliografía}

Banco Mundial (2005), World Development Indicators, Banco Mundial, Washington.

Barreiro-Cavestrany, Fernando (2007), "Territorios virtuosos para el desarrollo humano. Competitividad, cohesión social y ciudadanía en el desarrollo local", II Encuentro Latinoamericano Retos del Desarrollo Local. Gestión Innovadora de Territorios, Quito, 2023 de noviembre.

Conapo (Consejo Nacional de Población) (2005), Proyecciones de la población de México 2000-2050, Conapo, México.

Das, Tarun Kanti (1993), "Undp Human Development Index - Some Methodological Issues and Alternative Measures", mimeo.

Dávila, Enrique, Georgina Kessel y Santiago Levy (2002), El sur también existe: un ensayo sobre el desarrollo regional de México, Instituto Mexicano del Seguro Social, México.

Dirección Nacional de Planeación, Programación y Presupuesto-SeP (2004), Sistema educativo de los Estados Unidos Mexicanos. Principales cifras, ciclo escolar 2003-2004, Secretaría de Educación Pública, México. 
Dirección de Estadísticas e Información en Salud (2005), Estadisticas vitales. Información básica 2004, Ministerio de Salud y Ambiente de la Nación, Buenos Aires.

Hanham, Alison, Sam Berhanu y Scott Loveridge (2000), A Human Development Index for West Virginia Counties, West Virginia University, Virginia.

Herrero, Carmen, Ángel Soler y Antonio Villar (2004), Capital humano $y$ desarrollo humano en España, sus comunidades autónomas y provincias 1980-2000, Instituto Valenciano de Investigaciones Económicas, Valencia.

Hicks, Norman y Paul Streeten (1979), "Indicators of Development: The Search for a Basic Needs Yarstick", World Development, 7 (6), McGill University, Quebec, pp. 767-580.

INDEC (Instituto Nacional de Estadísticas y Censos) (2004), Microdatos de la EPH 2003-2010, INDEC, Buenos Aires, <www.indec.gov.ar>, 12 de agosto de 2008 .

INEGI (Instituto Nacional de Estadísticas, Geografía e Informática) (2004a), Estadisticas Vitales, Serie Boletin de Estadísticas Continuas, Demográficas y Sociales, INEGI, México.

INEGI (Instituto Nacional de Estadísticas, Geografía e Informática) (2004b), Encuestas en hogares. Encuesta Nacional de Ingreso y Gastos de los Hogares y Encuesta Nacional de Empleo, México, <http:// www.inegi.org.mx/inegi/default.aspx?s=est $\& \mathrm{c}=10205>, 3$ de septiembre de 2008.

Kelley, Allen (1991), “The Human Development Index: 'Handle with care", Population and Development Review, 17 (2), The Population Council, Nueva York, pp. 315-324.

López-Calva, Luis y Roberto Vélez Grajales (2003), "El concepto de desarrollo humano, su importancia y aplicación en México", Estudios sobre Desarrollo Humano, 1, PNUd, México, pp. 1-46.

Morris, David (1979), Measuring the World's Poor: The Physical Quality of Life Index, Pergamon Press, Nueva York. 
Murray, Christopher (1991), "Development Data Constraints and the Human Development Index", Discussion Paper 25, United Nations Research Institute for Social Development.

Nordhaus, William y James Tobin (1973), “Is Growth Obsolete?", Cowles Fundation Discussion Papers, 319, Yale University, Connecticut.

OCDE (Organización para la Cooperación y el Desarrollo Económico) (2002), Estudio territorial de México, ocDE, México.

PNUD (Programa de la Naciones Unidas para el Desarrollo) (1990), Desarrollo Humano: Informe 1990, Tercer Mundo Editores, Bogotá.

PNUD (Programa de la Naciones Unidas para el Desarrollo) (1991), Desarrollo Humano: Informe 1991, Tercer Mundo Editores, Bogotá

PNUD (Programa de las Naciones Unidas para el Desarrollo) (1990-2006), Human Development Report, Oxford University Press, Oxford.

PNUD (Programa de las Naciones Unidas para el Desarrollo) (1996), Desarrollo humano en Chile 1996, PNUd, Santiago de Chile.

PNUd (Programa de las Naciones Unidas para el Desarrollo) (2000), Desarrollo humano 2000. Más sociedad para gobernar el futuro, PNUD Santiago de Chile.

PNUD (Programa de las Naciones Unidas para el Desarrollo) (2002), Informe sobre desarrollo humano 2002, PNUD, México.

PNUD (Programa de las Naciones Unidas para el Desarrollo) (2003), Informe sobre desarrollo humano 2003. Los Objetivos de Desarrollo del Milenio: un pacto entre las naciones para eliminar la pobreza, Mundi Prensa, Madrid.

PNUD (Programa de las Naciones Unidas para el Desarrollo) (2004a), Informe nacional de desarrollo humano Bolivia 2004. Índice de desarrollo humano en los municipios de Bolivia, PNUD, La Paz.

PNUD (Programa de las Naciones Unidas para el Desarrollo) (2004b), Informe sobre desarrollo humano 2004, PNUD, México. 
PNUd (Programa de las Naciones Unidas para el Desarrollo) (2005), Informe de desarrollo humano 2005. Un tiempo de oportunidades. Argentina después de la crisis, PNUD, Buenos Aires.

PNUD (Programa de la Naciones Unidas para el Desarrollo) (2006); Informe sobre desarrollo humano. Más allá de la escasez: poder, pobreza y la crisis mundial del agua, Mundi Prensa, Madrid.

Qizilbash, Mosaffar (2002), On the measurement on Human Development, School of Economic and Social Studies, UEA, Norwich.

Ram, Rati (1982), "Composite indices of physical quality of life. Basic needs fullfilment and income. A Principal Component Representation", Journal of Development Economics, 11, London School Of Economics, Londres, pp. 227-247.

Ravallion, Martin (1997), "Good and Bad Growth: The Human Development Reports”, World Development, 25 (5), McGill University, Quebec, pp. 631-638.

Red Federal de Información Educativa (2006), Anuario Estadístico Educativo 2004, Ministerio de Educación, Ciencia y Técnica de la Nación, Buenos Aires.

Rofman, Alejandro y Luis Romero (1998), Sistema socioeconómico y estructura regional en la Argentina, Amorrortu, Buenos Aires.

Rofman, Alejandro (2006), "El enfoque del desarrollo local: conflictos y limitaciones", en Adriana Rofman y Alejandro Villar (comps.), Desarrollo local: Una revisión critica del debate, Espacio Editorial, Buenos Aires, pp. 37-58.

Sen, Amartya (1971), "Choice Functions and Revealed Preference”, Review of Economic Studies, 38, Stockholm University, Estocolmo, pp. 304-317.

Sen, Amartya (1987a), Hunger and Entitlements: Research for Action, World Institute for Development Economics Research of the United Nations University, Helsinki.

Sen, Amartya (1987b), Food, Economics and Entitlements, World Institute for Development Economics Research, United Nations University, Helsinki. 
Sen, Amartya (1988), “The Concept of Development”, en H. Chenery y T. Srinivasan (eds.), Handbook of Development Economics, vol. 1, Elsevier Science Publishers, North Holland, pp. 10-26.

Sen, Amartya (2000), Desarrollo y libertad, Planeta, Barcelona.

Slottje, Daniel (1991), "Measuring the Quality of Life Across Countries", The Review of Economics and Statistics, 73 (4), MIT Press, Cambridge, pp. 684-693.

Stanton, Elizabeth (2007), "The Human Development Index: A History", Working Papers Series, 127, University of Massachusetts, Amherst.

Ul Haq, Mahbub (1995), Reflections on Human Development, Oxford University Press, Oxford.

Ul Haq, Mahbub (2003), "The Birth of the Human Development Index", Readings in Human Development, Oxford University Press, Oxford, pp. 127-137.

Vaca, Josefina (2004), "Articulación regional y desarrollo desigual en el territorio argentino", Revista Territorios, 10-11, Universidad de Los Andes, Bogotá, pp. 111-125.

Recibido: 28 de noviembre de 2008.

Reenviado: 15 de mayo de 2010. Aceptado: 10 de septiembre de 2010.

María Albina Pol. Es doctora en economía por la Universidad de Alicante. Actualmente labora en la Facultad de Ciencias Políticas y Sociales como docente de grado y posgrado en la Universidad Nacional de Cuyo, Argentina. Ha sido becaria posdoctoral del Consejo Nacional de Investigaciones Científicas y Técnicas (Conicet), Ministerio de Ciencia, Tecnología e Innovación Productiva, Argentina. Es investigadora miembro del Programa I+D: "Desarrollo Local y Vitivinicultura 2005-2009" (Sectyp, Universidad de Cuyo). Sus líneas de investigación actual son: desarrollo local, desarrollo humano territorial y sistemas de indicadores. Entre sus últimas publicaciones se encuentran: en coautoría, "Los sistemas productivos locales desde la perspectiva del desarrollo humano", en Antonio Martínez Puche et al. (coords.), Los sistemas locales de empresas y el desarrollo territorial: evolución y perspectivas actuales en un mundo globalizado, Club Universitario, Alicante, pp. 151-166 (2008). 